\title{
Trends in radar absorbing materials technology
}

\author{
K J VINOY and R M JHA* \\ Computational Electromagnetics Lab, Aerospace Electronics \& Systems \\ Division, National Aerospace Laboratories, Bangalore 560017, India
}

MS received 28 October 1994

\begin{abstract}
The research in the area of Radar Absorbing Materials (RAMs) has been actively pursued for at least four decades. Although resonant RAMs were originally designed by transmission line approach, and the broad band RAMs were obtained by multilayering, the quest for ultrawide band performance has led to novel approaches such as chirality and even exploring biochemical products. It is observed that radome materials are frequently used as RAMs. The understanding of the underlying principles of electromagnetic analysis and design, fabrication and the trends in RAMs reviewed in this paper could lead to indigenisation, and even pioneering next generation of RAM technology.
\end{abstract}

Keywords. Radar absorbing materials (RAM); Radar cross section (RCS) reduction.

\section{Introduction}

The introduction of microwave radar, during the Second World War revolutionised the contemporary air defense scenario. The use of microwaves, enabled radars to detect even distant aircraft targets, independent of weather and diurnal variations. This provided the initial motivation for designing aircraft which would be "invisible" to radar. Since the late 1970 s, research efforts in this direction have intensified considerably.

Radar signal strength, reflected from a target, determines its detectability. This depends on the shape and size of the target and it is identified as its Radar Cross Section (RCS). Hence, the detectability of a target can be decreased by reducing its RCS. The RCS reduction is in fact important in many military and civitian applications. For the military, the RCS reduction of targets such as aircraft and missiles in hostile terrain is an important consideration. Similarly, making aircraft hangars near an air traffic control radar less reflective is a civilian application.

\footnotetext{
* For correspondence
} 
Several techniques have been suggested for RCS reduction. They are:

(i) Shaping,

(ii) active loading,

(iii) discrete (passive) loading, and

(iv) distributed loading.

Shaping involves modifying the external features of the scatterer to reduce the reflections, usually in the backscatter direction. Although shaping appears to be an excellent method for RCS reduction, it has several limitations as well. Normally reduction is achieved only in a limited angular region and at the expense of increased RCS in other regions. Furthermore, shaping is effective only at higher frequencies; in fact, at lower frequencies, it is almost ineffective. Finally, shaping must be integrated in the system design stage, right from the beginning; it cannot be implemented as an afterthought (Knott et al 1985).

Methods (ii) and (iii), employ active/passive elements at selected points on the target (where the reflections are maximum), to reduce the overall RCS by means of phase cancellation. But these are narrow bandwidth methods, still mostly of academic interest.

The distributed loading technique (iv) is considered to be the most useful and universal one. It consists of covering the scatterer with suitable materials called the radar absorbing materials (RAMs). The RCS reduction in this case is achieved by both, absorption and redirection of electromagnetic energy. Ideally, RAM should be an ultrawide band paint, effective at all polarisations, and angles of incidence. For application to flying objects, it should also have high tensile and compressive strengths, resistance to impact, tolerance to fuels and hydraulic fluids, and most importantly, low density (Bhattacharyya \& Sengupta 1991).

In order to increase their utility, suitable methods must be found to design broad band absorbers with narrow band RAMs as constituent components. Better still would be to search for alternative materials which have (ultra) wide band absorption characteristics. The answers to these are not readily available, owing to this being a classified area.

Although, almost all the literature on RAM is indeed classified, it is worth mentioning that historically, the origins of RAM have involved rather elementary principles in electromagnetic theory. Hence, considerable amount of relevant literature is also available in the open domain beginning from the late thirties. A closer scrutiny of this literature could give clues even to the RAMs that have been classified. Such a study could also give a feel of the historical perspective and the trends in RAM.

The historical perspective to the evolution of RAMs, including their basic performance characteristics is presented in $\$ 2$ of this paper. Fundamental electromagnetic principles for the analysis of $R A M s$, both on the flat and curvatured surfaces are outlined in $\S 3$. This is followed by the design and fabrication of RAM where both the resonant and broad band RAMs (including chirals) have been discussed. Since the characterisation of RAMs is extremely important from the design and development perspective, relevant measurement techniques have been introduced in $\S 5$. Although RAMs were primarily intended for RCS reduction of aerospace vehicles, they have found applications in several other areas as well; they have been comprehensively listed in $\S 6$. Finally, a review of the trends in $R A M$ is presented in $\S 7$. 


\section{The evolution of RAMs}

Although the commercial production of RAMs started during the 1950s, the research on it was well underway even during the 1930s. The first absorber material to be patented was the one developed in the Netherlands in 1936 (Naamlooze Vennootschap Machinerieen 1936). This was a quarterwave resonant material in the $2 \mathrm{GHz}$ region, in which carbon black was used to achieve dissipation, and $\mathrm{TiO}$, to achieve a high dielectric constant.

As pointed out earlier, with the advent of radars during the World War II, the quest for absorbers intensified. Germany and the United States began to work on projects to develop such materials. It is interesting to know the contrast between the ultimate motives of the two countries; Germany was primarily looking for absorbers for radar camouflage, while in the US, the research was directed primarily toward absorbers that would improve the radar performance (Emerson 1973).

Two types of materials (MacFarlane 1945; Schade 1945) were successfully developed in Germany for use in submarines. The first, called Wesch material consisted of a semiflexible rubber sheet loaded with carbonyl iron powder, and was resonant around $3 \mathrm{GHz}$. The second material, Jaumann absorber was developed by a multilayer approach. This provided reflection coefficients, typically lower than $-20 \mathrm{~dB}$ over a wide band $(2-15 \mathrm{GHz})$ near normal incidence.

In the US, two types of absorbers, generally called HARP (Halpern-anti-radarpaint) were developed during the same time (Halpren \& Johnson), which offered a reflection reduction of $15-20 \mathrm{~dB}$ at resonance. The airborne version, MX-410, was actually akin to a paint which utilised artificial dielectric materials of high relative permittivity. The second type was a rugged shipboard version consisting of a high concentration of iron particles in a neoprene binder. As with the MX-410, this too could operate in the X-band with a typical thickness of 0.07 inch. A salient feature of this version was the broadening of absorption around the resonant frequency (Montgomery et al 1948).

A well-known absorber developed around the same time as HARP, was the Salisbury screen (Salisbury 1952). A resistive sheet placed at quarter wavelength from the scatterer, was spaced by a low dielectric material. Salisbury screens operate by resonant technique, and were widely used in earlier anechoic chamber designs.

Yet another resonant absorber in wide use, was the Dallenbach layer (Ruck et al 1970). It consisted of a homogeneous lossy layer on a metal plate. The thickness of the lossy layer was selected so as to match its input impedance with the intrinsic impedance of the free space.

As discussed above, the absorbers developed in earlier stages were mostly narrow band in nature. This necessitated research towards obtaining broad band RAMs. Probably the very first approach to be tried was a multilayer structure (c.f. Jaumann absorbers discussed above). Most of the practical broad band RAMs were constructed using this technique. For example, the broadening of bandwidth of Salisbury screens was achieved by introducing additional layers of resistive sheets and spacers (Emerson 1973).

In some applications bulk absorbers were also used in place of sheets/layers. The conductivity of the bulk material reduces upon infusion of carbon in a matrix of spongy urethane foam. Based on this approach, the US Naval Research Laboratory developed a broad band absorber in the early 1950 s. The conductivity was reduced here, by 
dipping or spraying carbon onto a mat of loosely spun animal hair. These bulk absorbers could be readily used for controlling reflections for both, the indoor (anechoic) as well as the outdoor antenna measurement ranges.

During the late 1950s the focus shifted to problems of anechoic chamber implementation, such as (i) development of RAMs with reflection coefficients of -40 to $-60 \mathrm{~dB}$, (ii) lowering the operational frequency limit, and (iii) improved measurement techniques for RAM/anechoic chamber.

Attempts were also made to modify the resonant absorbers. For example, circuit analog RAM (CA-RAM) were obtained as Salisbury screen derivatives. CA-RAMs were obtained by depositing lossy material in geometric patterns on a thin lossless film (Severin 1956; Ruck et al 1970). The thickness of the deposit controlled the effective resistance of the layer, while the geometry of the pattern controlled its effective inductance and capacitance. Thus, the layer could be tailored into any value of impedance and its characteristic analysed in terms of lumped elements. The advantage of the CA-RAMs was that better performance could be obtained with comparatively less thickness.

In the 1960 's, the possibility of using magnetic materials as RAM was explored intensively. Thin magnetic $R A M s$ (e.g. the ferrites) with low reflection coefficients were fabricated which could operate at lower resonant frequencies than had earlier been feasible with the dielectric materials (Bowman 1968). Although thinner compared to the dielectrics, these materials are much heavier and prone to disintegration at higher frequencies.

It came to light in the 1980 s by serendipity, that some biotech products have ultrawide band absorption characteristics. Subsequent experiments confirmed that substantial RCS reduction was indeed possible by dissolving these compounds in aircraft structural materials. Following this spectacular revelation, the Department of Defense, US, immediately classified the compound. There are, however, conjectures that this ultrawide band $R A M$ is a retinyl compound belonging to the class of Schiff-base salts. This is a powdery black substance which is much lighter $(1 / 10)$ than the ferrites; it is also said to have been used in making RAM paints for stealth aircraft (Adam 1988).

More recently, a new class of homogeneous materials, making use of the chiral property have also found applications as RAMs (Jaggard \& Enghetta 1989). Such chiral RAMs depend upon their optical activity and circular dichroism, and the backscatter RCS is independent of polarisation. As compared to the dielectric and magnetic RAMs discussed above, the chiral RAMs have vastly superior RCS reduction characteristics (Jaggard et al 1990, 1991).

\section{Approaches to the analysis of RAM}

The radar absorbing materials are characterised by their electromagnetic(EM) properties such as the permittivity $\varepsilon$, and the permeability $\mu$. These are usually normalised with respect to the corresponding free space quantities $\left(\varepsilon_{0}, \mu_{0}\right)$.

$$
\begin{aligned}
& \varepsilon_{r}=\varepsilon / \varepsilon_{0}, \\
& \mu_{r}=\mu / \mu_{0} .
\end{aligned}
$$

The relative permittivity $\varepsilon_{r}$ and the relative permeability $\mu_{r}$ are in general complex 
quantities, denoted by

$$
\begin{aligned}
& \varepsilon_{r}=\varepsilon_{r}^{\prime}+j \varepsilon_{r}^{\prime \prime}, \\
& \mu_{r}=\mu_{r}^{\prime}+j \mu_{r}^{\prime \prime},
\end{aligned}
$$

where $\varepsilon_{r}^{\prime}$ and $\mu_{r}^{\prime}$ are the real components and $\varepsilon_{r}^{\prime \prime}$ and $\mu_{r}^{\prime \prime}$ are the imaginary components of the dielectric constant and the relative permeability of the medium respectively. The imaginary components account for the losses in the medium. $\varepsilon_{r}^{\prime \prime}$ depends on the conductivity of the medium, and $\mu_{r}^{\prime \prime}$ on the intensity of magnetisation. Equations (3) and (4) are usually written for convenience as

where

$$
\begin{aligned}
& \varepsilon_{r}=\varepsilon_{r}^{\prime}\left(1+j \tan \delta_{e}\right), \\
& \mu_{r}=\mu_{r}^{\prime}\left(1+j \tan \delta_{m}\right),
\end{aligned}
$$

$$
\begin{aligned}
& \tan \delta_{e}=\varepsilon_{r}^{\prime \prime} / \varepsilon_{r}^{\prime}, \\
& \tan \delta_{m}=\mu_{r}^{\prime \prime} / \mu_{r}^{\prime} .
\end{aligned}
$$

In (7) and (8), $\tan \delta_{e}$ and $\tan \delta_{m}$ are known as the electric and magnetic loss tangents and are used to express the losses in the medium. Materials having large values for their dielectric loss tangents are generally called dielectric materials, while those with large $\tan \delta_{m}$, the magnetic materials.

Both the dielectric and the magnetic materials could be used in the fabrication of RAMs. Although the loss mechanism in these differs, both absorb the EM energy and convert it into heat. Since the distances from the transmitting radars are usually large, this absorption of energy may not heat up the material much. But absorption is not the only mechanism by which RAMs operate. The reflections at the $R A M$ free space interface could be controlled by properly designing the coatings.

Two important parameters that dictate the propagation of waves through a medium are its refractive index $n$, and the characteristic impedance $Z$. In terms of the fundamental EM parameters, these are defined as

$$
\begin{aligned}
& n=\left(\mu_{r} \varepsilon_{r}\right)^{1 / 2}, \\
& Z=[\mu / \varepsilon]^{1 / 2}=Z_{0}\left[\mu_{r} / \varepsilon_{r}\right]^{1 / 2},
\end{aligned}
$$

where $Z_{0}$ is the intrinsic impedance of the free space. This is a constant $(=377 \Omega$ ) and is independent of frequency. It is clear from (9) and (10) that both $n$ and $Z$ are complex quantities.

\subsection{Analysis of RAMs on flat surfaces}

The method most commonly used for the analysis of a flat surface coated with a RAM is the transmission line approach. In this approach, the RAM is expressed as a lossy matching network between the free space and the scatterer which is represented in terms of a complex impedance.

Reflections occur when the EM wave travels from one medium to another. This reflection is represented by a term called the reflection coefficient $r$. In the case of a wave entering into a large flat surface from free space,

$$
r=\left(Z_{1}-Z_{0}\right) /\left(Z_{1}+Z_{0}\right)
$$

where $Z_{1}$ is the input impedance at the boundary surface. 
Thus the condition for zero reflection is:

$$
Z_{1}=Z_{0}
$$

Equation (12) requires that the surface input impedance be independent of frequency, and real. The input impedance for a wave entering at normal incidence at the interface with free space is given by

$$
Z_{1}=Z \tanh \gamma d
$$

where $Z$ is the characteristic impedance of the RAM defined in (10), $d$ is the thickness of the RAM,

$$
\begin{aligned}
& \gamma=(j \omega \mu(\sigma+j \omega \varepsilon))^{1 / 2}, \\
& \omega=2 \pi f,
\end{aligned}
$$

$\gamma$ being the complex propagation constant of the medium, $\sigma$ the electrical conductivity of the medium, and $f$ the operational frequency.

The reflection coefficient of a coated body is affected by the interference of the waves reflected at the surface of the RAM and at the metal-RAM interface. Their phases depend on $\varepsilon, \mu$ and $d$ while their relative amplitudes depend on the losses in the material (c.f. (13) \& (14)).

The input impedances for the parallel and perpendicular polarisations are different for oblique incidence. At an angle of incidence $\theta$ with respect to the normal, $Z_{1 \text { para }}$, the input impedance for the parallel polarisation is given by:

$$
Z_{1 \text { para }}=Z \cos \theta \tanh (\gamma d \cos \theta),
$$

and $Z_{1 \text { perp }}$ for the perpendicular polarisation is defined as

$$
Z_{1 \text { perp }}=[Z \tanh (\gamma d \cos \theta)] / \cos \theta .
$$

The corresponding reflection coefficients are

$$
\begin{aligned}
& r_{\text {para }}=\left(Z_{1 \text { para }}-Z_{0} \cos \theta\right) /\left(Z_{1 \text { para }}+Z_{0} \cos \theta\right), \\
& r_{\text {perp }}=\left(Z_{1 \text { perp }} \cos \theta-Z_{0}\right) /\left(Z_{1 \text { perp }} \cos \theta+Z_{0}\right),
\end{aligned}
$$

where $r_{\text {para }}$ is the reflection coefficient for parallel polarisation and $r_{\text {perp }}$ is the reflection coefficient for perpendicular polarisation.

Thus knowing $\varepsilon_{r}, \mu_{r}$ and $\sigma$ of the material, the input impedance can be determined by substituting (1)-(4), (10) and (13) -(15) in (11). Similarly by using (16)-(19) the reflection coefficient corresponding to an angle of incidence $\theta$ can also be determined (Ono \& Suzuki 1967).

Multilayer absorbers having broad band absorption characteristics can also be used for the RAM applications. Their analyses are similar to those of the single layer RAMs. The input impedance of the entire substrate, upon inclusion of an additional layer, depends on the properties of all the previous layers. A numerical program for computing the characteristics of multilayered RAM using a minimisation method is presented by Maffioli (1970). Ono \& Suzuki (1967) computed the reflection coefficient and the attenuation for both the perpendicular and parallel polarisations for multilayered RAM structures using the transmission line approach. The measurements on actual samples (Ono et al 1967) showed their method to be viable. 


\subsection{Analysis of curved surfaces coated with RAM}

The foregoing discussion is valid for the RAMs on flat surfaces in general. Analysis and design of coatings on curved surfaces, in contrast, is extremely complex.

3.2a Theorems on absorbers: Two theorems introduced by Weston (1963) facilitate the analysis of RAM on curvatured surfaces.

Theorem 1. If a plane EM wave is incident upon a body consisting of material such that $\mu_{r}=\varepsilon_{r}$, then the far zone backscattered field is zero, provided that the direction of incident propagation is parallel to an axis of the body about which a rotation of $90^{\circ}$ leaves the shape of the body together with its material medium invariant.

Theorem 2. If a plane wave is incident upon a body consisting of a material such that the total tangential EM field components satisfy the impedance boundary condition

$$
\mathbf{E}-(\mathbf{n . E}) \mathbf{n}=Z\left[\mu_{0} \varepsilon_{0}\right]^{1 / 2} \mathbf{n} \times \mathbf{H},
$$

on the surface, and the surface of the body is invariant under a $90^{\circ}$ rotation about some axis, then the far-field backscattered field is zero if the direction of incidence is along this axis. In the above expression $\mathbf{n}$ is the unit outward normal of the surface and $Z=1$ is the free space.

The above theorems are extended to predict the specular backscatter from any smooth arbitrarily convex bodies.

3.2b Effect of curvature: Bodies with curved surfaces in the high frequency domain (i.e. when they are electrically large) have two components for their backscatter, viz. the specular, and the diffracted field contributions. The specular contribution from an absorber covered body is $|r|^{2} \sigma$. Here $r$ is the flat plate reflection coefficient, and $\sigma$ is the specular reflection. The calculation for the diffracted field contribution is rather involved as it is affected by the shape of the scatterer, its conductivity and the direction of the ray with respect to the axis of the body. A detailed analysis is given by Weston (1963) and Bowman \& Weston (1966).

For extending these theorems to bodies in the resonance region (i.e. when their largest dimension is comparable to the wavelength), additional conditions must be satisfied by these curved surfaces. These ensure that, the creeping wave contribution is also reduced considerably for a general smooth convex scatterer. The boundary condition defined by the second theorem is a very good approximation under the assumptions (Leontovich 1957):

(i) The refractive index of the material is very large and has a large imaginary part.

(ii) The EM fields outside the boundary vary slowly in the near field, exterior to the scatterer.

(iii) The radii of curvature of the surface are large compared to the interior wavelength (i.e. corresponding to the wavelength of EM wave propagating inside the scattering medium).

(iv) Variations of $\varepsilon$ and $\mu$ in the body over the distance of the interior wavelength are small. This condition is relaxable for thin coatings on metallic surfaces. 
To summarise, an absorber with a large and complex refractive index significantly reduces the backscattered RCS in the resonance region of arbitrary convex bodies for the resonant frequencies where the main contribution to scattering is from the specular reflections and the creeping waves.

\subsection{Approaches to analysis of $R A M s$ on a general surface}

In the EM scattering and diffraction problems, the method of moments (MOM) presents one of the most versatile approaches. MOM is well known to predict accurate solution for the class of problems it treats. The applicability of MOM and other grid based approaches to the RAM analysis have therefore been explored by various workers. Rogers (1986) applied MOM to the RCS prediction of arbitrarily shaped conducting bodies coated with RAMs. MOM was also modified to effectively analyse the shapes used in the construction of anechoic chambers. Yang et al (1992) have developed a periodic moment method (PMM) for the analysis of two dimensional lossy dielectric scatterers. CRAY-YMP was used to calculate the reflection and transmission coefficients of periodically distributed wedges illuminated by a plane wave.

The grid-like segmentation of the MOM is also employed in the spatial network method by Kashiwa et al (1990). The scattering body, an aircraft, is divided into a number of cells of size $\lambda / 30$. In this time domain method, the equivalent circuit of the coated scatterer is constructed in terms of bulk impedances to compute the field patterns.

Yet another time domain analysis conforming to the Bergeron's method in three dimensional space and time is used to analyse resonant RAMs (Aoto et al 1987). The variation of field distribution for the changes in magnetic loss is given. Absorption characteristics of pulse waveform in the time domain are expressed as a function of the incidence angle.

Finally, the Finite Difference Time Domain (FDTD) approach is used in conjunction with the Levenberg-Marquardt nonlinear optimisation routine for forward scattering computations of broad band absorbers (Strickel \& Taflove 1990). The computational results for RCS reduction of two-dimensional targets are also provided in the paper. An earlier work on the lines of FDTD by Holland \& Cho (1986) reported reasonable accuracy (within 10\%) for RAM-coated cylinders even though only coarse grids were used.

The state-of-the-art in MOM and time domain methods, including the FDTD is such that it can yet handle only low frequency scatterers. However, with the trend towards use of ever increasing frequency (typically in $\mathrm{GHz}$ range) most of the realistic objects are essentially high-frequency scatterers. For such electrically large scatterers, solutions have originally existed in the form of Physical Optics (PO) (Macleod 1989) and Geometrical Optics (GO). Analyses of electrical large objects coated with RAMs have been mostly treated through the GO.

Moreland \& Peters (1966) applied GO to obtain an expression for RCS analyses of spherical and cylindrical bodies coated with dielectric shells. They considered both the specular and the creeping wave contributions and concluded that the latter gave rise to errors in the case of comparatively smaller bodies, while its effects are negligible for larger bodies. Earlier, a similar problem had been attempted using semiempirical methods to RCS computations of the spherical and cylindrical scatterers (Swarner $\&$ Peters 1963). The scattered field was approximated as the phasor sum of the field 
scattered by the air-dielectric interface and that by the equivalent conducting body which differs from the actual body because of the lens action of the shell.

Alexopoulos (1969) applied GO to obtain the reflected electric field for large spheres ( $k a=50$ to 1000 ) coated with inhomogeneous dielectrics. A series solution was also obtained for the backscattering GO computations for bodies of revolution coated with homogeneous RAMs (Arsaev 1982). The effect of the curvature of the scatterer and the conditions for the RCS reduction were also examined.

Although the GO is extremely simple to apply, it is well known to give rise to singularities and also predict discontinuous fields. This was overcome by the introduction of a class of high-frequency asymptotic theories which essentially introduced corrections to the GO in the form of diffraction coefficients. The Geometrical Theory of Diffraction (GTD) is one such example which has been extremely popular and also extensively modified to suit the various modelling requirements. Consequently, the asymptotic theories have found their way into the analysis of RAMs as well.

The RCS reduction of structures such as a cavity absorber has been pioneered by Lee et al (1985) for coatings on circular waveguides. They presented a high frequency asymptotic method to analyse EM scattering from circular waveguides coated with lossy materials. It was observed that a coating less than $1 \%$ of the radius can give $13 \mathrm{~dB}$ reduction in the RCS of circular waveguide terminated with a perfect electric conductor. It was also found that large reductions in RCS are possible at small angles of incidence.

The asymptotic high frequency techniques could also be employed for the estimation of monostatic RCS of finite planar metallic structure coated with a lossy dielectric. The RCS is expressed in terms of the diffraction coefficient involving the Maliuzhinet's function (Bhattacharyya \& Tandon 1984). Bhattacharyya (1990) used the uniform asymptotic theory of diffraction (UAT) which is a modification of the GTD, to analyse the performance of flat plates.

More recently, Shi et al (1993) have analysed conducting bodies covered partially with absorbent materials. A salient contribution of this work is in establishing an equivalence between the RAMs to be used in the scale model and the prototype.

\section{Design and fabrication of RAMs}

The design of RAMs involves identifying suitable materials, and specifying their dimensions and compositions. Similarly, the steps involved in the fabrication of these materials are crucial to their performance.

There are basically two concepts that are used in the design of absorbers for the RCS reduction (Musal \& Hahn 1989) as given below.

Matched characteristic impedance concept: The characteristic impedance of the material is made nearly equal to the intrinsic impedance of free space. This essentially means that the values of its relative permittivity and relative permeability must be equal.

$$
Z=Z_{0}
$$

hence from (10),

$$
\varepsilon_{\mathrm{r}}=\mu_{\mathrm{r}}
$$


Equation (22) is the condition for zero reflection at the front surface of the material. The material must be made sufficiently thick so that the EM wave is attenuated considerably as it propagates into the material. The internal reflections produced when backed by a metallic surface attenuate in the round-trip path.

Matched wave impedance concept: The wave impedance at the front surface of a metal-backed absorber layer (the impedance when looking into it) is made equal to the intrinsic impedance of the free space. This results in the complete absorption of the EM energy falling on the surface.

Thus for an absorber of finite thickness backed by a conducting plate, (13) gives the wave impedance. Combining (12) and (13), the thickness is determined as,

$$
Z_{0}=Z \tanh \gamma d
$$

Thus the formulae(1)-(19) can be used appropriately for computing the thickness of the RAM required for a specified performance. Knott (1979) has derived the formulae for the minimum thickness condition, for a specified reflectance of a single layer absorber, of given characteristic impedance and refractive index.

The matched wave impedance concept is frequently used in the design of the resonant absorbers. Although such resonant RAMs require comparatively thin layers of absorber, they satisfy the conditions for RCS reduction only at one or more discrete frequencies. On the other hand, the nonresonant absorbers have RCS reduction over a broader band of frequency. Hence these constitute the two basic types of RAMs.

\subsection{Resonant RAM}

The thickness of the resonant i.e. narrow band RAMs are related to the material properties and the operating wavelength.

4.1a Salisbury screen: The resonant absorbers discussed in $\$ 2$, strive to efface the reactive part of their surface impedance by suitably incorporating the unique properties of quarter wavelength. The simplest method is to make use of a resistance card, whose surface resistance is equal to the free space impedance. When sheet is placed at a quarter of a wavelength from the metal plate, the input impedance in the plane of the sheet and the impedance of free space are in perfect match. Such absorbers are called Salisbury screens (figure 1). A detailed analysis of the Salisbury screen is available in Ruck et al (1970).

The equivalent circuit for the Salisbury screen is shown in figure 2. The conditions to be satisfied by the resistive sheet are:

$$
\begin{array}{cc} 
& \varepsilon_{r}^{\prime \prime} \gg \varepsilon_{r}^{\prime}, \\
& Z_{0}=1 / \sigma d, \\
& d=1 / Z_{0} \sigma,
\end{array}
$$

where $d$ is the thickness of the resistive sheet. The choice of the material for the sheet is constrained by (24) while its thickness is determined from (26).

A proprietary material, Uskon cloth manufactured by the US Rubber Company was used as the resistive sheet in the Salisbury screens. Materials of relative permittivity 


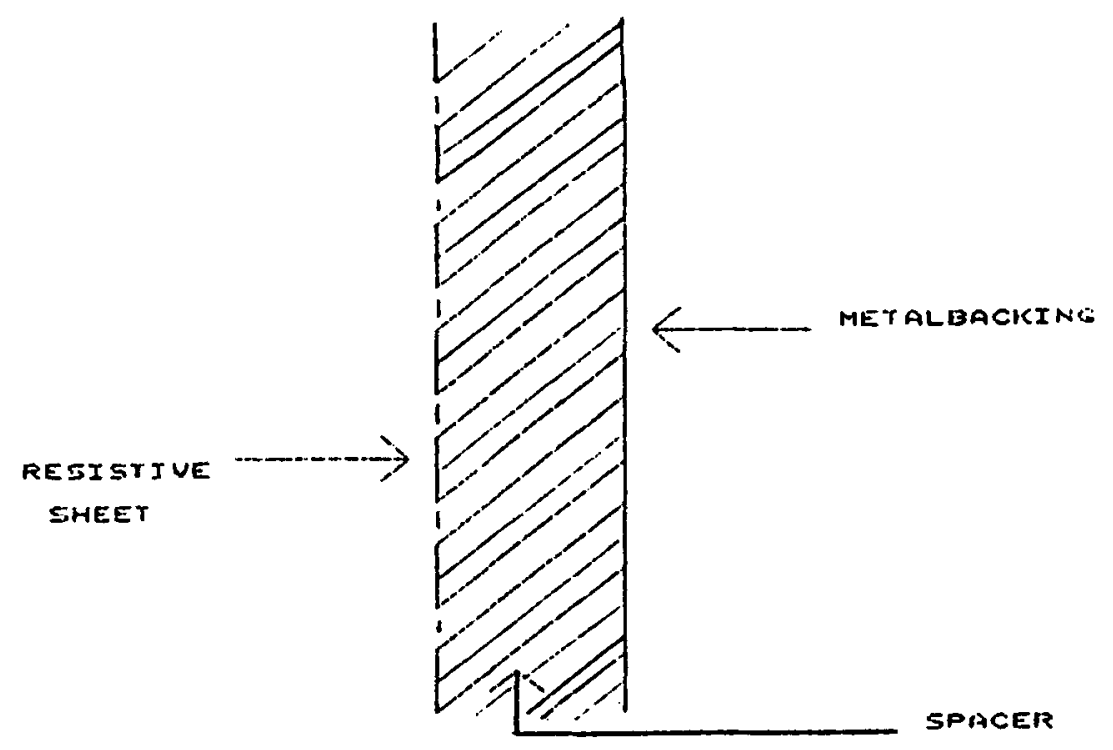

Figure 1. The Salisbury screen.

approximately equal to unity, were used as spacers. The operational bandwidth is reduced if the spacer material has a higher permittivity, as the wavelength inside it, is a function of this value. Plywood or some jelly type material having $\varepsilon_{\mathrm{r}}<1.05$ can be used as spacer. In one such case, the calculated frequency sensitivity for VSWR was found to be below 1.10 (that is, the reflection coefficient of 0.05 ) over a bandwidth of $\pm 5 \%$ (Emerson 1973).

The operational frequency range for the Salisbury screen can be broadened by using a number of such layers. Fante \& McCormack (1988) describe the analysis for Salisbury screens using multiple resistive sheets. The cases in which the screen is electric or magnetic or their multiple combinations are discussed. It is also shown that a relatively large reduction in specular reflection is possible over considerably wide bandwidths by using relatively few Salisbury screens. Furthermore, these reductions can be achieved

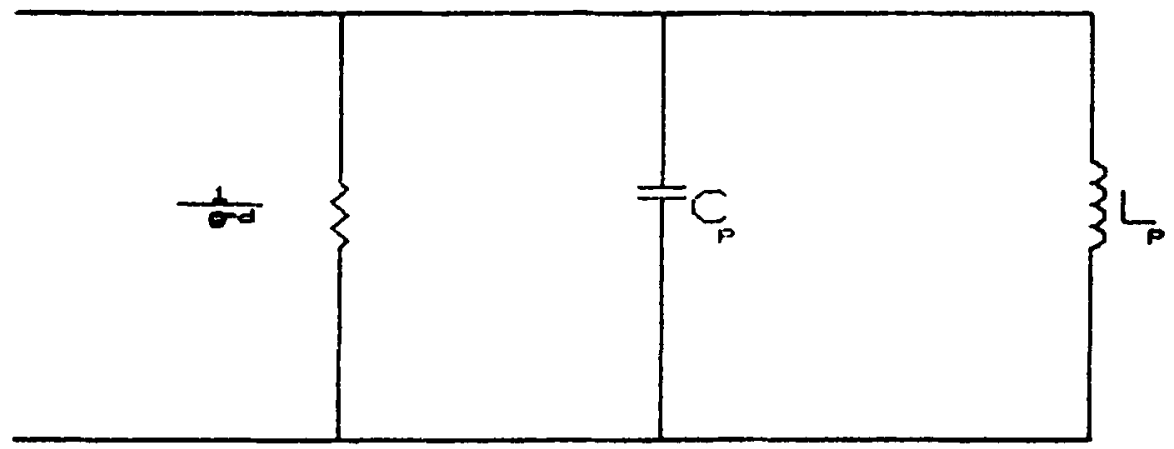

Figure 2. The equivalent circuit of the Salisbury screen. 
even if the incident wave is not normal to the surface, or the surface is curved, or the fabrication is not accurate.

A multilayer Salisbury screen uses several layers of Salisbury screens, suitably spaced to resonate at discrete frequencies, which results in broad band RAMs. The bandwidth of such a structure depends on the number of sheets used. Their construction is similar to the Jaumann absorber. The resistance of these sheets decreases near-exponentially towards the back plate.

4.1b Magnetic absorbers: The electric Salisbury screens place the lossy material where the electric field is maximum. It is also possible to place a magnetic screen where the magnetic field is maximum, that is, right on the metal surface.

The necessary conditions to be satisfied are:

$$
\begin{aligned}
& \mu^{\prime \prime} \gg \mu^{\prime}, \\
& Z_{0}=\omega \mu^{\prime \prime} d, \\
& \beta d \cos \theta \ll 1 .
\end{aligned}
$$

If a material with $\varepsilon, \mu$ and $d$, satisfies (13) along with (27)-(29) at the frequency of interest, it may be selected for fabrication. When the thickness is very small compared to the wavelength (29), the conditions (from (13)) to be satisfied by these materials are (Naito \& Suetake 1971):

i.e.,

$$
\begin{aligned}
& \mu_{r}^{\prime}=0, \\
& \mu_{r}^{\prime \prime}=\lambda / 2 \pi d \gg 1,
\end{aligned}
$$

$$
d=\lambda / 2 \pi \mu_{r}^{\prime \prime} .
$$

Typically these designs result in thicknesses of the order of $\lambda / 10$. Thus the magnetic materials have an edge over their dielectric counterparts as they require less thickness for a required $\mathrm{RCS}$ reduction.

In the magnetic absorbers, the losses are due to the presence of carbonyl iron or ferrites embedded in rubber. Small dipoles are present in these materials which try to orient themselves along the incident field. If the field changes fast, the dipoles lag the impressed field variations, and torque is exerted thereby dissipating energy in the material.

Most of the magnetic RAMs employ ferrites as the dissipating element. Each ferrite has two matching frequencies and two matching thicknesses which are related to an intrinsic constant of the material called the Snock's value (Naito \& Suetake 1971). The ferrite for a particular application is selected in such a way that the first matching frequency coincides with the operating requirements. The first matching thickness is independent of the frequency, and normally it is $8 \mathrm{~mm}$. This peculiar characteristic of ferrite is due to its magnetisation mechanism (Naito 1970). Three mechanisms for magnetisation are possible in a ferrite, namely, the relaxation magnetisation, the resonance motion of magnetic domain and the spin resonance motion. The first among these is dominant for the EM absorption.

Magnetic RAMs are prone to disintegration and their magnetic properties tend to deteriorate when ambient temperature reaches the Curie point (typically $500-1000^{\circ} \mathrm{F}$ ). Although ceramic ferrites can be used at higher temperatures, they are constrained by an upper frequency limit. 
The permeability of the ferrites depends on their composition. The peaks of the permeability curves of such materials can be shifted over frequency by replacement of $\mathrm{Fe}^{+3}$ ions in the lattice by divalent and tetravalent metal ions such as $\mathrm{Co}^{+2}$ and $\mathrm{Ti}^{+4}$ (Amin \& James 1981). Cobalt-substituted barium hexaferrite has significant variation of permeability in the microwave region. The ferromagnetic resonant frequency of this hexagonal ferrite varies between 2 and $46.5 \mathrm{GHz}$ depending on the amount of cobalt substituted.

The ferrites are typically sintered in the form of small rigid tiles. The density of these tiles depends upon the composition and the method of processing (i.e. different temperatures and pressures applied during manufacture). The surface resistance can be varied during manufacture by suitable selection of molecular structure and loading concentration. The material is hot-pressed onto the metallic surface at several thousands of psi pressure. Bonding of the materials is also a crucial process. It is thus possible to obtain magnetic RAMs with different characteristics by slightly varying the fabrication procedure (Hahn 1991). The effects on the magnetic permeability of different $\mathrm{Ni}_{0.1} \mathrm{Zn}_{0.5} \mathrm{Fe}_{2} \mathrm{O}_{4}$ samples are studied in a number of such cases. It is observed that the real and imaginary parts of permeability increase with the tile density.

The commonly used binding media are natural rubber or synthetic elastomers such as isoprene, neoprene, nitrile, silicone, urethane, and fluoroelastomer. Although the EM absorption properties of the binding media normally remain constant, there are instances where they behave anomalously. It is reported (Veinger et al 1990) that at low filler contents, low molecular weight rubbers such as dimethyl-phenyl-siloxane rubber, dimethyl-diphenyl-siloxane rubber, and dimethyl-siloxane rubber showed strong absorption near $10 \mathrm{GHz}$.

High frequency absorption of a random assembly of noninteracting single domain ferromagnetic particles with positive uniaxial magnetic anisotropy can be calculated numerically for values of the signal frequency below the natural resonant frequency of such materials (Hempel \& Roos 1981). If the lower frequencies are used, an additional absorption is found to be present at the negative field at which the resonance frequency crosses the signal frequency. A set of measurements has also been specifically reported by Hempel \& Roos (1981) for the polycrystalline barium ferrites.

Just as their dielectric counterparts, the magnetic RAMs too can be used in multilayered forms. Basically, this is done to enable the employment of materials that have favourable permeability curves at different frequencies.

4.1c Dallenbach (homogeneous) layer: Yet another dielectric RAM, the homogeneous absorber typically consists of a binding medium (e.g., polyurethane foam) in which carbon powder and $\mathrm{TiO}$ are dispersed. The amount of carbon determines the dissipation factor, while the $\mathrm{TiO}$ content accounts for the high permittivity thereby resulting in a thinner layer.

The design for the absorber is generalised by Fernandez \& Valenzula (1985). They considered the case for a single layered absorber backed by a perfectly conducting flat surface for normal incidence. Substituting (5), (6) and (10) in (13) one obtains

where

$$
\begin{aligned}
& \left.\mu_{r} / \varepsilon_{r}=\left\{(\cosh 2 \beta d s+\cos 2 \beta d) \cos \delta_{m}\right)\right\} /\left\{(\cosh 2 \beta d s-\cos 2 \beta d) \cos \delta_{e}\right\}, \\
& \sin 2 \beta d=p \sinh 2 \beta d s,
\end{aligned}
$$

$$
s=\tan \left[\left(\delta_{m}+\delta_{e}\right) / 2\right],
$$




$$
\begin{aligned}
& p=\tan \left[\left(\delta_{m}-\delta_{e}\right) / 2\right] \\
& \beta=\left\{2 \pi\left(\mu_{r} \varepsilon_{r}\right)^{1 / 2} \sin \left[\left(\delta_{m}+\delta_{e}\right) / 2\right]\right\} /\left\{\lambda\left(\cos \delta_{e} \cos \delta_{m}\right)^{1 / 2}\right\}
\end{aligned}
$$

The design curves for different conditions are obtained from (33) and (34). These lead to the solution for $d$ as:

$$
\begin{array}{ll}
d=\pi /[2 \beta(1+p s)], & p<0 \cdot 25, \\
d=(1 / 2 \beta)[6(1-p s) /(1+p s)]^{1 / 2}, & p \geqslant 0 \cdot 25 .
\end{array}
$$

The quarter-wavelength solution is obtained by substituting $p=0$ in (38).

The lowest order solution for the materials having both electric and magnetic losses, is given in terms of plots by Fernandez \& Valenzula (1985). They have also concluded that the materials having moderate losses will have a larger bandwidth for absorption.

An alternate solution along with optimal design curves for the EM and physical parameters such as the surface impedance, and magnetic and dielectric loss tangents, and the thickness in wavelength is given(Musal \& Hahn 1989) for the normal incidence. Design curves are plotted for different $d / \lambda$ and $\tan \delta_{e}$.

A further generalisation by Musal \& Smith (1990) resulted in an integrated single design curve which is plotted as contours of constant $d / \lambda \operatorname{Re}(\varepsilon)$ and contours of constant $\operatorname{Im}(\varepsilon) / \operatorname{Re}(\varepsilon)$ on a plane of $(d / \lambda \operatorname{Im}(\mu), d / \lambda \operatorname{Re}(\mu))$.

Yet another example for the homogeneous absorbers is the Halpern Anti-Radar Paint (HARP). Disk-shaped aluminium flakes dispersed in a rubber matrix serve as the dissipating elements in HARP. High concentration and a high degree of alignment of these disks resulted in a large permittivity (Knott et al 1985).

A new kind of single layer absorber postulated by Gauss (1982) could be made from filaments of radar absorbing chaff mixed in a solid binder. Metallic strands of 50 micrometers length could be dispersed in the medium having a dielectric constant of about unity at spacings of about one-third their length. Theoretically, RCS reduction of about $30 \mathrm{~dB}$ is achievable over $10-100 \mathrm{GHz}$ if the filaments are distributed uniformly.

Multiple layers of the homogeneous absorbers, each with a different composition could also be used to obtain broad band absorbers. In practice, this results in an inhomogeneous absorber to be discussed later in $\$ 4 \cdot 2 \mathrm{c}$.

4.1d Circuit analog RAM: If the continuous resistive sheets of the Salisbury screens are replaced by a conductive layer deposited in appropriate geometrical patterns, circuit analog RAMs (CA-RAM) are obtained. These shapes, usually in the form of loop antennas, dipoles, crosses or triangles may have susceptance as well as conductance, which improves the flexibility of the design of such RAMs.

The design of these patterns is similar to that of a frequency selective surface (FSS). But CA-RAM is different from the FSS since the latter does not require resistance variation. Both involves the design of frequency sensitive reactive elements.

Control over admittance of the conductive sheet in CA-RAM is obtained by depositing the conducting materials in suitable geometrical shapes. The shapes employed have the property of resonating at a discrete frequency, thus dissipating the energy falling on it. Typical shapes include strips, wires, intersecting wires, dipoles, crossed dipoles, Jerusalem cross structure etc. These sheets with the lossy elements are separated from the conducting surfaces by lossless dielectric spacers. 


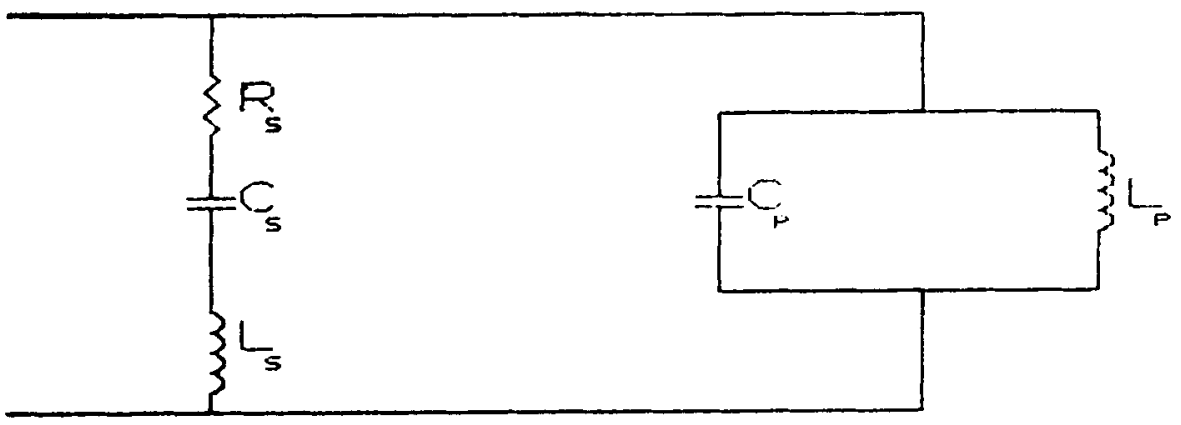

Figure 3. The equivalent circuit of the CA-RAM.

The equivalent circuit of the CA-RAM is shown in figure 3. By adjusting the shape of the elements, and/or by adjusting the number of elements per unit area, the resonance, and the zero reflection may be obtained at any frequency. The optimum values for both of these are selected. It is clear that a better uniform loading is obtained when the elements are in the molecular level; i.e., by using magnetic dipoles in molecules.

For a periodically loaded surface, the input impedance at oblique incidence, for the parallel and perpendicular polarisations (i.e., $E$ parallel and perpendicular to the plane of incidence respectively) are:

$$
\begin{array}{ll}
Z_{1 \text { para }}=X \cos \theta & \text { (parallel polarisation) } \\
Z_{1 \text { perp }}=X / \cos \theta & \text { (perpendicular polarisation), }
\end{array}
$$

where $X$ is the element impedance and $\theta$ the angle of incidence. The reflection coefficients are obtained by substituting these expressions in (18) and (19) (Ruck et al 1970). Consequently, at normal incidence $\left(\theta=0^{\circ}\right)$, the impedances and hence the reflection coefficients for the two polarisations are identical.

The absorber is effective only if the magnetic vector of the incident wave has a component in the direction of the magnetic dipoles. Hence two separate grids with arrays of elements in parallel, with an angle of orientation of $90^{\circ}$ between the grids, each for a polarisation, may be used to make a RAM independent of polarisation.

\subsection{Broad band absorbers}

Unlike the resonant absorbers discussed above, the broad band absorbers have a wider band of frequency over which considerable RCS reduction is possible. This band of frequency is usually limited only by the changes in the material properties.

4.2a $\mu=\varepsilon$ absorbers: Thick layers of materials satisfying the condition $\mu_{r}=\varepsilon_{r}$ are used in the first type of nonresonant absorbers called the $\mu=\varepsilon$ absorber. In these RAMs most of the incident energy is absorbed by the material itself, before reaching the metal plate at the back. Thus for a material with both dielectric and magnetic losses (Severin 1956),

$$
Z=\left[\mu_{0} / \varepsilon_{0}\right]^{1 / 2}\left[\mu\left(1-\tan \delta_{m}\right) / \varepsilon\left(1-\tan \delta_{e}\right)\right]^{1 / 2} .
$$

For the matching condition with free space, the characteristic impedance of the 
material should be:

$$
Z=Z_{0}=377 \Omega \text {. }
$$

An efficient $\mu=\varepsilon$ absorber should have large and equal values for dielectric and magnetic loss angles, and relative permittivity and permeability. Ideally, these EM parameters should also be independent of frequency.

The electric and magnetic field strengths are attenuated in the bulk of the material exponentially. Thus the minimum thickness for a specified attenuation is determined from (Hurmuth 1983)

or,

$$
\left[E_{r} / E_{i}\right]=e^{-2 \beta d},
$$

$$
d=(1 / 2 \beta) \ln \left[E_{r} / E_{i}\right],
$$

where $E_{r}$ and $E_{i}$ are the reflected and incident fields respectively.

Although absorbers with $\mu_{r}$ equal to $\varepsilon_{r}$ are ideal, this is one of the most difficult conditions to meet in fabrication. The EM properties of the materials depend on the composition and are in general frequency sensitive. Some ferrites have been made with this constraint for a limited frequency range (Bhattacharyya \& Sengupta 1991).

4.2b Low density absorbers: The low density absorbers are made with the materials with relative permittivity nearly equal to that of free space. If the relative permeability is also 1, the material will absorb all the energy falling on it. Most of the materials satisfying these conditions are materials of low density. Thick layers of these materials are used to dissipate the energy, thus reducing the reflections when backed by a metal plate. For analysis and design purpose, this may be considered as a special case of the $\mu=\varepsilon$ absorber.

One such material manufactured, Spongex was 2 inches thick, and had a reffection coefficient of about $-20 \mathrm{~dB}$ over a frequency range of $2 \cdot 4-10 \cdot 0 \mathrm{GHz}$. It was possible to further lower the (low) frequency limit by increasing the material thickness (Emerson 1973).

4.2c Inhomogeneous layer absorber: The absorbers should present a minimum discontinuity at the free space-absorber interface and the losses should increase, as one moves into it, to absorb the incident EM energy completely. This is obtained either by tapering of homogeneous layers of absorber material, or by arranging different layers parallel to the metal surface such that the loss tangent increases toward the base plate. The latter results in an inhomogeneous absorber. In another method of construction, layers of lossless dielectric and sheets of poorly conducting material, the surface resistivity of which decreases towards the base metal plate by a constant factor from one sheet to the next, are placed alternately. Theoretical predictions for one such case shows that reflection is below $0 \cdot 1$ for nearly 3 octaves (Bowman 1968). However, these values are not achievable in practice, since it is difficult to fabricate a number of layers having different parameters accurately.

In a bulk absorber with a multilayered structure, the EM properties of different layers are made to vary for obtaining maximum absorption of the incident energy. The thickness of the bulk material is not important. The refractive indices of the layers are such that the value increases as one goes into the material. A simple fabrication technique, where the foam is dipped in an aqueous solution of graphite, the aquadag, of different concentrations, is described by Jones \& Wooding (1964). The injection of carbon is made uniform by alternately compressing and expanding the foam inside the 
solution. Yet another multilayer absorber was fabricated by making use of pyrolysed polyacrylonitrile fibre blankets for different layers (Ono et al 1967).

It is possible to synthesise an inhomogeneous absorber with a number of layers with different composition of carbon using the convergence circle method (Ono et al 1979). The propagation constant is related by an empirical relation to the graphite density in the media. The thickness of the material as well as the required concentration of the graphite content in a polystyrene foam for a given angle of incidence and figure of merit have been computed. (The figure of merit is defined (Shimizu \& Suetake 1969) as the ratio of the RAM thickness to the wavelength of the lowest frequency at the maximum angle of incidence.) These wide incidence angle response absorhers are reported to have very good reflection properties $(-20 \mathrm{~dB})$ at normal as well as at oblique incidences up to \pm 70 .

Analytical determination of the functional form of the variation of $\mu(z)$ and $\varepsilon(z)$ necessary to produce a specified maximum reflection coefficient over a range of frequencies and incidence angles for a particular thickness of the material is an intractable problem. In practice, some model variations are assumed for $\mu(z)$ and $\varepsilon(z)$ to analyse the material (Ruck et al 1970; Perini \& Cohen 1991).

Naito \& Suetake (1965) used a relay impedance method to design a multilayered absorber which was primarily meant for anechoic chambers. With the input impedance at the metallic wall being zero and the intrinsic impedance of free space $377 \mathrm{ohms}$, the absorber layer is essentially a transformer between these. When multiple layers are used, obviously, this transition is achieved in steps. Naito \& Suetake (1965) have computed the frequency response of absorbers with different number of layers and plotted this for comparison. These formulae were later modified (Shimuzu \& Suetake 1967) for the optimum thickness using lossy dielectric materials. They have reported a figure of merit of 0-42 as compared to the earlier designs, for which it was nearly 2 .

4.2d Geometric transition absorhers: The difficulty in making multiple layers with different values of surface resistivity can be bypassed by using a single layer of uniform material in geometrical shapes in which the absorption coefficient increases towards the metal surface. Wedge. pyramidal, and conical shapes are typically used for this purpose. Such shaped absorber panels are widely used in the wall construction of anechoic chambers. However, these are not used in airborne applications due to obvious aerodynamic considerations. The geometric transition method can also be combined with the inhomogeneous layer method to further increase the bandwidth of the absorber. Combining these two techniques, results in a better angular performance. Typically, for an inhomogeneous pyramidal absorber, the reflection coefficient is below $0 \cdot 1$ for incidence angles up to $5060^{\circ}$ (Ruck et al 1970).

This section is concluded by emphasising that the broad band absorbers cannot be used by themselves, for aircraft and missile applications due to their inherent bulkiness. However, when mixed with structural materials, composites are obtained having broad band RCS reduction characteristics.

\subsection{Chiral materials}

The materials discussed so far are in general composites, and are sometimes even anisotropic in nature. The design and the manufacture of these materials are often 
Table 1. Comparison of characteristics of simple isotropic and chiral media (Jaggard \& Engheta 1989).

\begin{tabular}{lll}
\hline Characteristics & \multicolumn{1}{c}{ Simple medium } & \multicolumn{1}{c}{ Chiral medium } \\
\hline Constitutive relations & $D=\varepsilon E$ & $D=\varepsilon E+j \zeta B$ \\
& $H=(1 / \mu) B$ & $H=j \zeta E+(1 / \mu) B$ \\
Wave equation & $\nabla \times \nabla \times E-\beta^{2} E=0$ & $\nabla \times \nabla \times E-\beta^{2} E-2 \omega \mu \zeta \nabla \times E=0$ \\
Wave numbers & $\beta=\omega[(\mu \varepsilon)]^{1 / 2}$ & $\beta_{c \pm}= \pm \omega \mu \zeta+\left[\beta^{2}+(\omega \mu \zeta)^{2}\right]$ \\
Wave intrinsic impedance & $Z=[(\mu / \varepsilon)]^{1 / 2}$ & $Z_{c}=Z /\left(1+Z^{2} \zeta^{2}\right)$ \\
Eigen modes & Linear polarisation & Only right and left circular \\
& allowed & polarisation allowed \\
\hline
\end{tabular}

difficult. Their EM properties are frequency-dependent and hence they are usually narrow band. In contrast, a new class of materials based upon EM chirality (handedness) when used as RAMs (Jaggard \& Engheta 1989), offer an increased bandwidth along with improved reflection coefficient characteristics.

Chirality combines optical activity (rotation of the plane of polarisation) and circular dichroism (change in polarisation ellipticity) in the medium which is expressed by two unequal wave numbers corresponding to the two circularly polarised eigen modes with opposite handedness. These materials are isotropic as well as reciprocal. The fundamentals of chirality are available in literature (Post 1962; Kong 1975). The constitutive relationships and characteristics of such media are compared with simple isotropic media in table 1.

In table $1, \zeta$ is the chirality admittance which expresses the handedness of the medium, and affects the wave transmitted through the medium. In fact, $\zeta$ is a measure of the cross coupling of electric and magnetic fields in the medium. The twist in the major axis of the polarisation ellipse depends on the sign of $\zeta$.

The chirals consist of an isotropic host medium which is embedded with randomly oriented identical microstructures, such as the microhelices. The geometric dimensions and density of these chiral microstructures determine the EM parameters $\varepsilon, \mu$ and $\zeta$.

The absorption in chiral materials is determined by the chiral skin depth while their reflection properties are governed by $\varepsilon, \mu$ and $\zeta$. The backscattering in these materials is independent of polarisation. Hence the introduction of these chiral materials for Salisbury screens and Dallenbach layers, results in increased absorption over wider frequency band with the added advantage of thinner layers (Jaggard et al 1990). For the electric Salisbury screen, the surface impedance may be expressed as

$$
Z_{1}=j Z_{c} \cot \beta_{c} d
$$

while for the magnetic screen, it is given as

$$
Z_{1}=-j Z_{\mathrm{c}} \tan \beta_{c} d \text {. }
$$

Substituting these in (23), the thicknesses of the chiral layer for a required maximum reflection coefficient can be determined. Thicknesses of the order of $\lambda / 5$ give rise to 20 $\mathrm{dB}$ more absorption as compared to nonchiral materials (Jaggard et al 1990).

An EM pulse propagation analysis in the chiral media has shown that the chiral coatings can be used for broad band RCS reduction even in high power pulsed radar environment (Engheta \& Zablocky 1990). 
More recently, thin layers of biisotropic (nonreciprocal) chiral materials that can act as polarisation transformers with ultrawide range of frequency have been proposed by Tretyakov \& Oksanen (1991).

\subsection{The construction of $R A M s$ in practice}

Antenna mount structures, especially those employed in measurement ranges or chambers are fabricated by using nonreflective materials. Blore (1964) describes the fabrication and testing of four different polyfoam structures made for this purpose. The scattering mechanism for polyfoam includes the effect of cell size, material homogeneity, water content, surface finish and matching methods. The low echo area, mechanical rigidity and relative ease for manufacture and usage make these materials the best suited for antenna support.

Bradshaw (1989) has studied the effect of construction methods on the RCS reduction in structural materials such as syntactic foam, light weight honeycomb, single skin fibre reinforced composites (FRC) as well as the sandwich composites. The application of these materials having microwave absorption properties for the aircraft $\mathrm{RCS}$ reduction is quite apparent.

Broad band response becomes essential for RAMs used in radar targets in a wide band radar environment. The reflectivity curves of Eccosorb (NZ-series) materials suitable for such applications have been evaluated by Hurmuth (1983). Their performance is analysed for a relative bandwidth of about unity. Compared to the performance at a relative bandwidth of 0.01 , these absorbers are still less helpful in hiding targets from wide band radars (He et al 1992).

Some of the Eccosorb materials have good absorption properties even above $35 \mathrm{GHz}$. In particular, the CR-series Eccosorb materials have been characterised by Hemmati et al (1985). It is concluded that the measurements are in perfect match with the curves formed by extending the specifications for these materials at lower frequencies. The reflection and absorption coefficients increase with the density of iron in the material. It is also found that the absorption coefficient decreases by a factor of two, when the samples are cooled to cryogenic temperatures.

Ferrites or carbon may be interspersed in rubber to make absorbers for covering the edges of paraboloidal reflectors (Yokoi \& Fukumaro 1971). The antenna performance is improved by as much as $10 \mathrm{~dB}$ at $70^{\circ}-180^{\circ}$. The addition of carbon increases the permittivity of acetylene black rubber, with the change in the imaginary part being more predominant; theoretical computations for the same have been compared with the experimental results by Kumar (1987).

Aspects of modelling with spinel ferrites, magneto and dielectric composite materials for forming RAM are overviewed by Deleuze (1992). Ferrofluid composites containing mono layers of aluminium particles dispersed in a decalin carrier are considered by Davies et al (1986) for frequencies above $100 \mathrm{GHz}$. Chains of aluminium particles with oxide coatings are developed. It is observed that if the plane of polarisation is parallel to the chain axis, there is a minimum effect on the variation of frequency.

Ferrite sludge obtained by precipitation treatment of industrial waste water has been reported to possess microwave absorbing properties (McCauley et al 1980). The powder so obtained is sintered into a mechanically coherent ferrite ceramic material. Composites of ferrites and resins $9.5 \mathrm{~mm}$ thick are reported to operate as microwave absorbers. These composites are found to be operational in the S-band ( $68 \%$ composite) 
as well as in the X-band ( $75 \%$ composite), and are suggested as an inexpensive choice for $\mathrm{RCS}$ reduction.

A broad band absorber with $50 \%$ relative bandwidth is obtained with a two-layer ferrite-resin mixture incorporating short metal fibres. Materials of $4.6 \mathrm{~mm}$ thickness with fibres of 1-4 mm length and 60 micrometer diameter are found to be useful in the $\mathrm{X}$-band $(8-13 \mathrm{GHz})$. Good performance for wide angles of upto $45^{\circ}$ is also obtained for these materials (Hatakeyama \& Inui 1984).

The constitutive relations for the maximum absorption of EM energy falling on a medium has been presented by de Hoop (1981). Metal coated carbon fibres were also used as conducting elements in RAM (Yi \& Gan 1991). The use of such fibres results in improved attenuation and a wider bandwidth. The absorption properties are independent of the thickness of the material, but are related to fibre dimension and concentration.

Broad band microwave absorption cannot be achieved by homogeneous materials. However, it was noticed experimentally that some compounds made with small conducting grains dispersed in a dielectric host can be used as broad band RAMs (Guillot \& Bobillot 1991). Various mixture laws for such designs are developed by Guillot (1992) for the computation of dielectric permittivity. Mixtures, for which the quasistatic approximation is not matched inside the conducting medium, are considered. This results in artificial lossy diamagnetic behaviour for which a model is derived.

The application of chiral materials on spherical targets offers significant improvement in the reflection coefficient $(15-25 \mathrm{~dB})$ as compared to the conventional materials for the radii of curvature of the order of half a wa velength or larger (Jaggard et al 1991).

Polymers containing silicon are observed to possess superior electrical and mechanical properties and can be used for RCS reduction of aircraft. A fabrication process, in which electropolymerised film comprising such polymers or copolymers is deposited on the surface of an anode, is described by Nagasubramanian et al (1990). Conductive polymers like polypyrrolle, polyaniline and polyacrylthiophenes have also been reported in literature as materials in the fabrication of RAMs (Olmedo 1992).

\section{Absorber mieasurement techniques}

The measurements on RAMs are conducted either to survey or verify product performance and material properties. In this context, usually three forms of material are measured:

(i) Finished absorber panels or products,

(ii) Thin sheets used as components in the design and fabrication process,

(iii) Samples of uniform bulk materials.

A number of methods are explained in various books and papers on microwave measurements (Ginzton 1957; Montgomery 1957; Ono et al 1983; Knott et al 1985; Curran 1993). Some of the important methods are listed below.

- Parallel plate

- Coaxial probe

- Transmission line techniques

- Waveguide system
- Resonant cavity

- Admittance tunnel

- Free space techniques 


\subsection{Parallel plate method}

The parallel plate method requires that the material being tested is homogeneous, and nonmagnetic. The sample taken should be thin (less than $10 \mathrm{~mm}$ ), flat and perfectly smooth. An LCR meter or an impedance analyser measures the impedance across a pair of parallel plates separated by the sample. The errors in this low frequency (less than $100 \mathrm{MHz}$ ) method are mainly due to fringing fields at the edges. Guarded electrodes can be used to eliminate the edge capacitance.

The features of this method are as below.

- Broad band

- Simple and convenient
- Accurate and sensitive

- Inexpensive

The RAMs, though usually operative in the microwave region, can nevertheless be accurately characterised in the audio frequency region. Lynnworth (1964) observed that the insertion loss in the microwave region is correlated to the capacitance of the material at the audio frequencies. Avcoat and polyurethane foams have been tested by the parallel plate method successfully (Lynnworth 1964). Acrylic panels in which molybdenum wires of 1 mil diameter are embedded at varying spacings are used as the standard for establishing the correlation.

\subsection{Coaxial probe technique}

The coaxial probe technique is useful for isotropic, homogeneous and nonmagnetic materials. Samples of 'infinite' thickness with one flat surface, and without any air gap may be tested with this method. This method is best suited for liquids and inviscid fluids. The minimum thickness for solid samples is about $20 / \varepsilon_{\mathrm{r}}^{1 / 2} \mathrm{~mm}$ (Curran 1993).

The advantages of the method are:

- Broad band

- Simple and convenient

\subsection{Waveguide system}

Reflectivity measurements of finished products at lower frequencies (typically $100-400 \mathrm{MHz}$ ), can be performed by placing a test sample at the aperture of a waveguide. Since the measuring probe is not movable, adjustments are made at the attached shorting stub. Although only single frequency measurement is possible at a time, stepped frequency measurements is also possible with additional equipment. Swept frequency interferometer has been used with an aperture matched exponential horn for the rapid real time assessment of inhomogeneities in planar microwave absorber panels (Baker \& van der Neut 1988). The residual reflection in such a system is as low as $-37 \mathrm{~dB}$.

A circular waveguide can be used more effectively than coaxial cables for measuring the plane wave reflection properties of layered dielectrics (Rudduck \& Yu 1974). This method is applicable to media containing high refractive index absorbing materials. The measurement set up can be very easily calibrated and the method is obviously nondestructive. 


\subsection{Transmission line techniques}

The transmission line techniques require that the voltage reflection coefficient of a homogeneous material sample be measured. This is done by multiple measurements with different terminations using slotted section. The samples must be carefully machined so as to accurately fit inside waveguide or coaxial line without any air gaps and their faces should be flat, smooth and perpendicular to the line of axis. The thickness of the samples must be in the range $\lambda / 18$ to $\lambda / 2$. Periodic dropouts, when the sample thickness approaches $\lambda / 2$, is a major failure of the method. This method is suitable for anisotropic and magnetic materials.

In the case of coaxial cable environment, the diameter of the standard cable may not be big enough for a test sample to be placed inside. This problem is avoided by using a larger section of cable of about one inch diameter connected to the standard cables using suitable taper sections. Unambiguous determination of the electrical thickness of the sample necessitates multiple frequency measurements. The sample is backed by metal sheet (a short), or an open circuited termination. The changes in the standing wave pattern inside the line upon inserting the sample lead to the characterisation of the material.

The attractive features of the method include its being (i) broad band, and (ii) simple and convenient.

The transmission line technique using coaxial cables is accurate up to $10 \%$, whereas, the waveguide method is precise up to $5 \%$ if the samples are machined properly (to a precision of $0.03 \mathrm{~mm}$ ).

Although the transmission line techniques are useful to a great extent in characterising the RAM, they have some limitations as well. Better results are guaranteed by the SCOC and thin sample methods if some precautions are taken (Falkenbach 1965). The sample thickness should be of the order of $\lambda / 50$ for the thin sample method, while it should be $\lambda / 5$ for the SCOC method. It is preferred that the material sample have dc resistance of about $1 \mathrm{k} \Omega / \mathrm{mm}$ thickness.

Some of the algorithms used in computation of results from transmission line measurements are listed in table 2.

Table 2. A comparison of computational algorithms employed in transmission line methods (Curran 1993).

\begin{tabular}{|c|c|c|c|}
\hline Algorithm & Measures & Computes & Ideal for \\
\hline Nicolson-Ross & $\begin{array}{l}S_{11}, S_{12} \\
S_{21}, S_{22}\end{array}$ & $\varepsilon_{r}, \mu_{r}$ & $\begin{array}{l}\text { Magnetic, short \& lossy } \\
\text { samples; fastest computation } \\
\text { speed }\end{array}$ \\
\hline $\begin{array}{l}\text { Precision } \\
\text { (NIST) }\end{array}$ & $\begin{array}{l}S_{11}, S_{12} \\
S_{21}, S_{22}\end{array}$ & $\varepsilon_{r}$ & $\begin{array}{l}\text { Long low-loss samples; } \\
\text { highest accuracy if no } \\
\text { discontinuities present }\end{array}$ \\
\hline Fast & $\begin{array}{l}S_{11}, S_{12} \\
S_{21}, S_{22}\end{array}$ & $\varepsilon_{r}$ & $\begin{array}{l}\text { Faster and better method for } \\
\text { lossy materials than the } \\
\text { previous one }\end{array}$ \\
\hline Short-backed & $S_{11}$ & $\varepsilon_{\mathrm{r}}$ & Liquids and powders \\
\hline Arbitrary-backed & $S_{11}$ & $\varepsilon_{r}$ & $\begin{array}{l}\text { Thin films, and high } \\
\text { temperature applications }\end{array}$ \\
\hline
\end{tabular}


With swept signal sources or using time domain reflectometry technique, fast measurements are possible over a wide range of frequencies. The time domain reflectometry technique involves sending a narrow pulse waveform which is scanned by a computer controlled broad band sampling oscilloscope. This is transformed into the frequency domain by the fast Fourier transform (FFT). Selection of timing windows is crucial for the accuracy of the measurements. Improved stability of the pulse generators as well as the sharpness of the pulses result in more accurate performance.

\subsection{Cavity technique}

A part of the cavity resonator is filled with the sample without affecting the field distribution inside. This technique, essentially a single frequency one, involves complex analysis. But it is suitable for anisotropic materials as well. This is the most accurate technique, especially for low loss homogeneous materials and liquids. Tunable cavities are used in practice when multiple frequency measurements are required.

\subsection{Admittance tunnel method}

The admittance tunnel is a long absorber-lined box illuminated by a small antenna at one end and fitted with a test aperture at the other. A hybrid Tee is used to isolate the transmitted and received signals. A continuous wave (CW) signal source is used for transmission. Since the transmitting and receiving antennas are the same, the residual reflections could be minimised. The box shape is useful in obtaining a near planar incident wave with little disturbance from the outside environment. This method, developed by Rockwell International Corporation, is ideal for thin sheets.

\subsection{Free space techniques}

The free space techniques are in general high frequency, non-contacting and nondestructive methods, typically applicable to large, flat, parallel faced homogeneous samples. Notwithstanding the title, the measurements are not always performed in free space-like environment, but are not done inside the confines of a waveguide or a cavity. It is difficult to measure the loss of thin and low loss samples with these methods. In general, the errors in free space techniques are due to: (i) finite sample size, (ii) non-plane wave illumination (iii) mechanical instability or misalignment between sample and antenna, and imperfect quality of anechoic environment.

Typically, the accuracy of measuring $\varepsilon_{r}$ is $1-5 \%$ while the value of $\tan (\delta)$ is precise to \pm 0.005 . Some of the important methods under this category are listed below.

- NRL arch method

- RCS range method

- Tunnel method

- S-parameter method
- Interferometer method (open resonator)

- Direct spatial standing wave measurements

- Double horn-reflector system

5.7a NRL arch method: This near-field method, developed by Naval Research Laboratory (NRL), operates by illuminating the absorber panel by a small horn antenna. A second horn antenna, which is also mounted on the same arch-like 
framework, receives the reflected energy. This being a near-field method results in lower reflectivity values. Only the amplitude characterisation of the reflected signals is possible here. Using swept oscilla tors, the measurement over a range of frequencies can be taken (Lehto et al 1991).

5.7b RCS range method: The RCS range method is a far-field technique in which the material sample is mounted on a flat plate which is in turn installed upon a target support column in a vertical position. The panels are evaluated in a more realistic environment akin to the one in which they may actually be used. In order to accommodate swept frequency signal sources, dedicated costly instrumentation may be required. The size of the plate is selected to be greater than $3 \lambda$, typically, $5 \lambda$. Due to limitation in determining the null width, the maximum length of the edges of the sample should be less than $15 \lambda$.

5.7c Interferometer method: The nature of reflections from a sample measured outside the confines of a closed system can also lead to characterisation of EM properties of the material. This set-up is similar to that of transmission line technique $(\$ 5.4)$ but for the sample holder and sliding short, which are replaced by a tuner and a horn. The sample is mounted on a metal support plate, a few feet in front of the horn. The tuner is used to remove the residual reflections. At higher frequencies, this method is similar to the Fabri Perot Optical Interferometer technique, used in characterising optical materials. An open cavity is used at microwave frequencies.

The open cavity method has been used successfully to obtain the complex magnetic permeability of materials loaded with conducting powders (Guillot \& Bobillot 1991). The material characteristics are obtained by measuring the resonant frequency and the quality factor of the cavity when a slab of the sample is placed inside. This method is, however, not suitable for taking measurements at high temperatures.

5.7d Free space tunnel method: The sample in the form of a sheet is placed between transmitting and receiving antennas. The spacing on either side of the sample should be greater than $2 d^{2} / \lambda$, where $d$ is the largest dimension of the antenna or the sheet. The power transmitted through the sample is measured to determine its EM properties.

5.7e Free space S-parameter method: This is an accurate measurement method in which the $S$ parameters are measured both in amplitude and phase using sophisticated equipment such as a vector network analyser. Although the material characterisation is better, the capital expenses are high.

5.7f Direct spatial standing wave meastrement technique: The direct spatial standing wave measurement technique is used for precise characterisation of absorber panels, especially of pyramidal shape. An infinitesimal dipole antenna is moved about the panel and the field strength is measured at each position.

This method is suitable for measurements at oblique angles of incidence. Absorbers can be quickly characterised over a wide range of frequencies and incident angles with this method. The scattering parameters like VSWR and scatter angle have been measured for pyramidal absorbers using this method (Ono et al 1983). The error in the 
measurement is found to be $\pm 20 \%$ for VSWR and \pm 3 for scattering angle at $4 \mathrm{GHz}$, when measurements are taken $1.5 \mathrm{~m}$ away from the sample.

5.7g Double horn-reflector system: A new method of characterising rectangular panels of homogeneous RAMs have been suggested by Kent (1982). In this wide band technique, the permittivity and permeability of the materials are obtained from the measured values for $S_{11}$ and $S_{21}$. The accuracy of this frequency domain method is stated to be $5-10 \%$.

Of the methods discussed above, the NRL arch method is the most famous and the most frequently used. Although this method is universal, it has some disadvantages as well. The disadvantages of this method are listed and some novel free space methods are suggested by Brumely (1987). These are

(i) inverse synthetic aperture radar imaging for samples with large area;

(ii) wide band coherent radar measurements and;

(iii) modification to the conventional time gathered RCS measurements, which are made as a function of the aspect angle of the sample wall of the targets.

Yet another nondestructive technique for characterising absorber-lined chambers is to use short dipoles as the measuring probes (Mishra et al 1982). These dipoles are reported to have a discrimination of the order of $-25 \mathrm{~dB}$. The microwave goniometer could also be used for characterising absorber panels (Jones \& Wooding 1964).

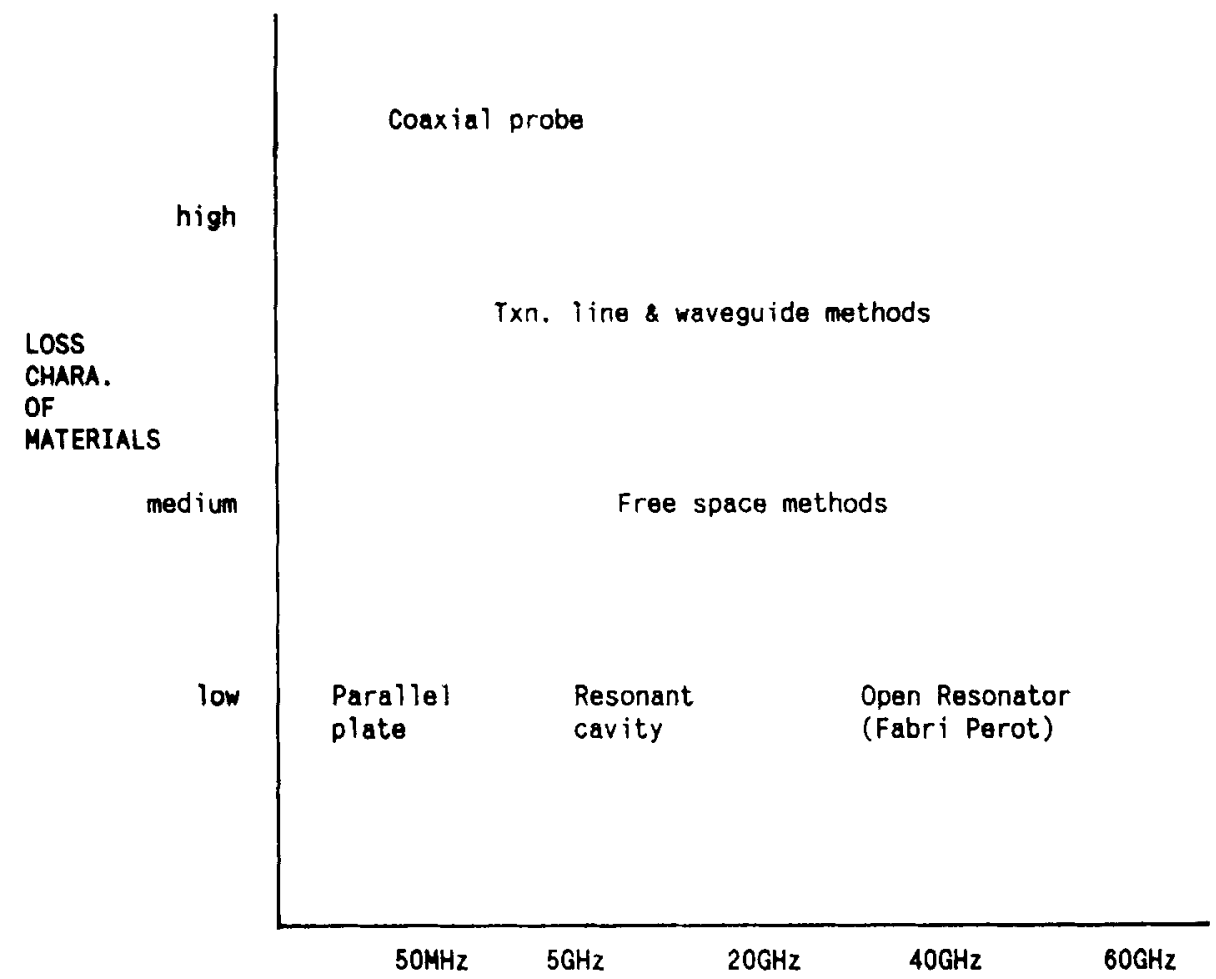

Figure 4. The suitability of the measurement methods for given frequency range and the nature of the material. 
Other novel methods also reported are microwave diversity imaging (Li et al 1989) and microwave scanning method (Wims \& Palmer 1991) which are essentially nondestructive in nature.

The frequency ranges of application of some of the measurement methods discussed above are given in figure 4 (Curran 1993). The optimum method for an application is chosen after taking the various limitations and advantages of these methods into consideration. Methods usually selected for different applications are listed below.

$\begin{array}{cl}\text { Product evaluation } & \begin{array}{l}\text { Free space techniques } \\ \text { Interferometer } \\ \text { Waveguide }\end{array} \\ \begin{array}{c}\text { EM properties of } \\ \text { bulk materials }\end{array} & \begin{array}{l}\text { Pareguide } \\ \text { Parallel plate } \\ \text { Transmission line techniques }\end{array} \\ \text { Sheet materials } & \text { Admittance tunnel }\end{array}$

Free space techniques

\section{Applications of RAM}

Although initially intended for camouflaging aircraft, and for enhancing radar performance, RAMs have found their way into numerous other areas. Some of the important applications of the RAMs are given below.

\subsection{RCS reduction of aerospace vehicles}

RAMs are primarily used to achieve broad band RCS reduction for aircraft (Walkington \& Huster 1979; Sweetman 1982, 1987; McCluggage 1987; Schmitman \& Warwick 1990; Cobucci 1991; Brown 1992; Martin 1992) and missiles (Hanson \& Kiehle 1982). Ferrite-based paints are one such class of materials developed earlier that were found to be useful, not only over wide band but also at the lower frequencies. Lighter RAMs were fabricated subsequently, but most of them are still on the classified list.

\subsection{Improvement of radar performance}

Absorber sheets are used to shield such reflecting objects in the vicinity of the radar that may otherwise cause spurious effects on radar signals at the base of the radome, parts of the antenna mechanism etc. In shipboard applications, the mast and the bulkhead are also covered with absorbers. Similarly, treating the buildings near an air traffic control radar with RAM, improves radar performance (Emerson 1973).

\subsection{Reduction of antenna $R C S$}

In some military applications it becomes necessary to keep the RCS of antennas as low as possible. Covering the aperture with an absorber coating can lead to considerable $\mathrm{RCS}$ reduction. In a typical case with a circular waveguide termination (Lee et al 1985), this $R C S$ reduction can be as much as $13 \mathrm{~dB}$. 


\subsection{Antenna sidelobe reduction}

The sidelobe level of antennas can be significantly reduced by placing the antenna within a tube made of absorber panels. Similarly obstructions from feed supports in reflector antennas are eliminated by covering them with absorber panels (Emerson 1973). The effect of edge diffraction in very large reflector antennas can be significantly reduced by covering the edges of the reflector with absorber sheets (Yokoi-\& Fukumaro 1971).

\subsection{Anechoic chambers}

Anechoic chambers are required in taking diverse EM measurements such as the aircraft RCS patterns, the antenna radiation patterns, the radome boresight error and various other simulation studies. An anechoic chamber for this purpose has to be isolated from the outside environment so that ideally no signal crosses the wall in either direction. The walls, floor and ceiling of such chambers are made absorptive to create a free-space-like environment.

The suitability and design of various RAMs for this application are widely reported in open literature (Severin 1956; Cherepanov 1974; Mitsmakher 1980; Brumely 1987; Joseph 1988). The design of broad band absorbers for electromagnetic susceptibility (EMS) measurements chambers was suggested by Mishra et al (1982).

Most of the chambers use absorbers cut in the pyramidal, conical or even an aggregate sine-wave shape for improved performance. Such chambers in the test region have maximum reflection levels $70 \mathrm{~dB}$ below the direct path signal, and shielding isolation as low as $-140 \mathrm{~dB}$ (Emerson 1973). State of the art anechoic chambers have a frequency range of about $100 \mathrm{MHz}-100 \mathrm{GHz}$.

\subsection{Improvement of outdoor range performance}

The effect of scattering from isolated objects in an outdoor range can be offset by covering them with RAMs. This results in a free-space-like environment so that the tests are more reliable. Polyfoam structures are found to be very effective in such applications (Blore 1964).

\subsection{EMI applications}

With the increasing use of higher frequencies in electronic circuits, it has become necessary to provide shielding from the electromagnetic interference (EMI) from/to nearby systems. Absorber-lined enclosures may be used when an ideal free space condition has to be met without disturbing the surroundings for electronic circuits. Customised absorbers are used in high power applications (Emerson 1973).

\subsection{As part of the microwave components}

A number of waveguide and coaxial microwave components make use of some absorbing material inside. For example, resistive vanes made of such materials are employed in 
making microwave attenuators (Nagornov et al 1978) and phase shifters (Tretyakov \& Oksanen 1991; Marty et al 1992). Absorber materials are also used for harmonic suppression in transmitters, and for gain stabilisation in amplifiers (Bostick 1985).

\subsection{Mode suppresser in circular waveguides}

When the inside surface of a circular waveguide is coated with RAM, the lower order modes are suppressed significantly. The amount of suppression depends on how lossy the material is. Such a mode of suppression can also result in the RCS reduction of circular structures. Coating the inside surface of a cavity with RAM reduces its RCS significantly (Lee et al 1985, 1986; Chou et al 1987; Chou 1988).

\subsection{In parallel plate waveguides}

When the inner surface of a parallel plate waveguide is covered with a RAM, it can propagate $T M(e)_{10}$ and $T M(e)_{10}$ modes over unlimited bandwidth. Such parallel plate waveguides are much more useful than a corrugated horn as a feed antenna. A detailed analysis of such structures is described by Cheng \& Ostertag (1986). It is shown that the performance is insensitive to the RAM characteristics.

\subsection{Protective shields in microwave oven}

A household application of RAM comes in the form of protective shields in microwave ovens. Ferrite paints are coated over the oven casings to reduce any possible leakage of the radiation to the atmosphere. A commercially available paint from the Japanese company TDK was effectively used for this purpose (Ashley \& Gilmore 1988).

\subsection{Removal of ghost images in TV pictures}

A paint from NEC could reduce the reflections significantly in the frequency range 3-5-20 GHz (Ashley \& Gilmore 1988). This sandwich ferrite RAM was successfully used for removing ghosts in TV pictures due to clutter effects from nearby buildings. Interestingly, in the early stages of the US Department of Defense stealth programme, this Japanese paint was in high demand.

\subsection{Electronic countermeasure applications}

Innovative applications of RAMs have also been proposed in Electronic Countermeasures $(E C M)$. A recent patent suggests coating the inner surface of countermeasure cartridge with plastisol (Tsuji 1992). The heat generated while expending the countermeasure payload softens the plastisol coating so that the entire container acts as a radar absorber rather than a reflector.

\subsection{RCS enhancement of the missile decoys}

Quite ironically, absorber materials can themselves be used to increase the RCS as well. In order to improve the detectability and hence the effectiveness of a missile decoy, its 
rear side is coated with absorbers (Harrington 1987). This reduces the creeping waves, thus resulting in increased $\mathrm{RCS}$ in the head-on, i.e. the backscattering direction.

\section{Trends in RAM}

As mentioned in $\$ \$ 1$ and 2 of this paper, contrary to the popular belief, RCS reduction is not one of the latest "emerging" technologies. The active research endeavor in this area is at least three, possibly four, decades old. The Soviets by 1965 had already noticed and were alarmed by the massive and increasing US funding to explore RCS reduction as an effective means of combat. The US interest and the intensification of the programme could be judged by the sharp funding increase by the US Air Force from 35.5 million (in 1962) to 119 million (in 1963) to 155 million dollars (in 1964) (Shneyderman 1985). Hence, the genesis for active research in RCS reduction can be traced back to the late Fifties rather than, as is popularly believed, to the early Seventies.

When the stealth programme was officially unveiled in the early Eighties, the cost of Raytheon B-2 was placed at 650 millions per piece (as compared to 15 million for the Lockheed F-16). As was later to be extensively commented upon by the experts in the aerospace industry, B-2 is highly inoptimal from aerodynamic considerations; its novelty lies in its stealth characteristics. Of these, shaping as a means for RCS reduction was known, and depended solely on enormous computer time for the stealth design. It is apparent from the external features of B-2, that it used smooth blended convex profiles as a means of shaping. It can therefore be presumed that much of this money for $\mathrm{RCS}$ reduction (B-2 is a 20 billion-dollar programme) went for the novel research on RAM technology.

A parallel exists in the Lockheed Have Blue and its successor F-117A models. From the shaping point of view, both the Have Blue and F-117A had a rather incongruous profile consisting of large external flat plates. The second means of achieving RCS reduction was to incorporate RAM in the aircraft structure itself. The cost of Lockheed $\mathrm{F}-117 \mathrm{~A}$ is once again prohibitively large.

Although US agencies have closely monitored the development of RAM technology in foreign countries, for example Russia and China, it has not been possible to put a cost tag to these efforts. In case of China, it is opined that much of the effort is being funded indirectly through the science and technology budget. It is conjectured that rather large RAM research programmes exist with the other four super powers, sometimes without even logical conclusion. For example, the French have displayed an extensive research interest in RAMs without apparently having a corresponding military aircraft programme.

It has indisputably emerged, from the figures connected with stealth programme, that the price of RAM technology has tended to be prohibitively high. Much of this research must have been directed towards analysis. design and development, fabrication and measurement aspects of RAM. We propose to look into the trends in RAM technology with this perspective.

\subsection{Bandwidth}

The EM absorbers made a rather modest beginning. It started with the quarterwavelength transmission line concept of impedance matching. A constraint in applying 
dielectric RAMs is their bulkiness. This could be offset by applying thin coatings of ferrites and other magnetic RAMs. However, both the dielectric and magnetic RAMs were frequency-dependent, and therefore narrow band absorbers.

The narrow band RAMs can be used in multiple layers to obtain broad band absorbers. However, either due to the bulkiness (dielectric RAMs) or higher density (magnetic RAMs), the weight penalty is still considerably high.

A new class of RAM based on the principle of EM chirality is reported to be much lighter than the ferrites and requires thinner coatings $(\lambda / 5$ to $\lambda / 6)$ than the conventional RAMs. Of late, a newer class of RAM based on Schiff-base compounds has been reported which is said to be as much as ten times lighter than the magnetic RAMs for the same absorptive performance. Furthermore, these retinyl derivatives (bacteriorhodopsin) are reported to have ultrawide band absorption characteristics.

\subsection{Analysis}

The analysis of RAM for future prediction has followed the developments in computational electromagnetics. In the high frequency domain, the methods initially used in the 1960s were geometrical optics (GO) and its extensions. The modification of GO to the GTD-type theories permitted RAM analysis of increasing complexity; these are discernible in the shooting and bouncing ray (SBR) method of Lee (1985) to analyse the RAM-coated engine cavity. The approach taken in the low-frequency domain has been that of the method of moments (MOM).

However, both GTD-type theories, and the MOM and its derivatives suffer from severe shortcomings. The GTD requires the scatterer to be convex, and the symmetrical cavities have stretched the SBR method to its limit. As of today, asymptotic raytheoretic methods cannot handle either a scatterer having concave and convex surfaces in the neighbourhood, or a surface of arbitrary concavity. The MOM can solve such problems with remarkable ease but the size of the scatterers must be electrically small. Unfortunately, the size (electrical) of the scatterer encountered in reality makes the application of MOM computationally intractable.

The time domain methods, (based on finite difference), which are similar to the MOM, have of late caused much interest. The finite difference time domain (FDTD) method was proposed in 1966 (Yee 1966). But it is only with the advent of parallel computers that its potential seems to have been unlocked. FDTD can yet solve only low-frequency scatterers, although at a speed usually one order of magnitude better than the MOM. Kashiwa et al (1990) have successfully presented a three-dimensional time domain analysis, of an electrically small aircraft structure coated with thin resonant absorbers. It is expected that, if the technological breakthroughs in parallel computers continue at the current pace, FDTD algorithms should be able to solve electrically large problems in another ten years time. In this context, it must be mentioned that the fastest supercomputers to date, require 10 days on stand-alone basis to analyse aircraft coated with RAM.

\subsection{Design}

Since most of the military and particularly the electronic warfare applications require wide bandwidths, it is essential that RAMs have broad band or even ultrawide band 
characteristics. The present practice is to obtain broad band RAMs by multilayering of resonant RAMs. In actual practice however determining the thickness of individual layers and the concentration gradients are highly computation intensive. Bastiere (1989) has applied fuzzy set theory to evolve a multi-criteria optimisation method for designing RAMs consisting of as many as 5 different layers.

\subsection{Measurements}

As mentioned in $\S 5$, well-known methods exist for characterising RAMs right from the audio frequencies up to $60 \mathrm{GHz}$ with varying degrees of accuracy. In contrast, Hemmati et al (1985) made transmittance and reflectance measurements on Eccosorb in the frequency range $35 \mathrm{GHz}-3 \mathrm{THz}$ at both ambient and cryogenic temperatures. The development of measurement techniques in the quasi-optical range has acquired additional significance, since the extensive use of this section of EM spectrum for next-generation military applications is imminent. Such developments tie well with the NASA Terahertz Technology Programme which is being funded on a priority basis.

\subsection{Fabrication}

Although applying RAMs to military aircraft structures is decidedly advantageous, it is somewhat offset by the weight penalty it imposes on the cruise performance of the aircraft. It is now feasible to use radar absorbing structures (RAS) in the construction of the aircraft. Since the RAS consists of embedding RAMs within the parts of the aircraft structure, there is almost no weight penalty. The concept of RAS is also consistent with the present day trend of increasing use of composites in the fabrication of fighter aircraft. In the state-of-the-art Lockheed F-18 aircraft (which interestingly has inherent stealth characteristics) the composites used are $9.9 \%$ by volume. In contrast, for the YF-23 ATF, a future aircraft programme, this percentage is to go up by as much as 45-50\% (Cobucci 1991).

RAS have been obtained by embedding magnetic RAMs in polymeric matrix (Stonier 1991). Similarly the use of bismaleide resins have also been reported in these composites. In the vicinity of engines, which is a major contributor to the overall RCS of the aircraft, polyamides have been used (Stonier 1991). Finally, novel fabrication techniques for RAS have succeeded in sustaining the RCS reduction characteristics at temperatures as high as $350^{\circ} \mathrm{F}$ which is well in excess of the maximum temperature encountered by the state-of-the-art supersonic fighter aircraft.

As a further corollary of the RAS concept, the possibility of constructing inherently stealth radomes is also being considered (Kumar et al 1994; Vinoy \& Jha 1994). Polyethylene fibres in composites result in RAS of high strength which can be used for EM window (radome) applications (Cain \& Corda 1991).

It is interesting to note that several radome material derivatives e.g., spinel ferrites (Deleuze 1992) have been successfully used as RAMs. This is hardly surprising since the electromagnetic analysis and design of radome and RAM involve similar physical parameters such as dielectric and magnetic loss tangents (Kumar 1994; Vinoy \& Jha 1994).

Since the exterior of the aerospace vehicles are subject to environmental hazards, the current trend is to develop RAMs which are inherently resistant to the environmental 
elements. Silicone rubber, which is a well-known anti-rain erosion material, has been tested as RAM (Army Material Development and Readiness Command 1982). Similarly RAMs have been developed with antiablation and nuclear-damage resistance properties. Inorganic ceramics $\mathrm{Al}_{2} \mathrm{O}_{3}$ and $\mathrm{ZrO}_{2}$ have been tested in this context. Once again, it may be worthwhile to recall that both these inorganic ceramics are extensively used in constructing hypersonic radomes for missiles (Kumar 1994; Kumar et al 1994).

\section{Summary}

The research in Radar Absorbing Materials (RAMs) as a viable means for RCS reduction has been actively pursued for at least four decades. The RAMs fabricated initially were based upon transmission line approach and were narrow band absorbers. The applicability of these resonant absorbers was enhanced by multilayering to obtain broad band absorbers.

Analyses have however shown that the ultrawide band radars can be effectively used as electronic countermeasures against broad band absorbers. This has motivated the current search for ultrawide band RAMs. Compared to the transmission line approach and multilayer concept, the initial successes reported in the area of ultrawide band RAMs have come from the completely novel approach of chirality and by exploring even biochemical products for this purpose.

In order to overcome the penalty of weight imposed by multilayered dielectric or ferrite RAMs coated on the aircraft structures, there is a decided shift towards using Radar Absorbing Structures (RAS) where the RAMs are embedded in the polymeric composite matrix. The concept of RAS is compatible with the trend of using increasing volumes of composites in the fabrication of the latest and of future generation fighter aircraft.

A comparison with our concurrent study in radomes has shown that several RAMs are actually radome materials. This observation is significant since the RAMs in general are classified, whereas extensive information on the radome materials is available in the open literature.

Besides the obvious military applications, RAMs have many prevailing and potential applications in the industrial and even domestic sectors. RAM has thus emerged as a typical dual-use technology, where commercially viable research and development can be pursued independent of the defense sector.

An awareness of the underlying principles of RAM analysis, design and fabrication methods, and the actual materials used as RAMs, could enable material scientists to indigenise and even pioneer the next generation of RAMs.

\section{References}

Adam J A 1988 How to design an invisible aircraft. IEEE Spectrum (4): 26-31

Alexpoulos N G 1969 Radar cross section of perfectly conducting spheres coated with a certain class of radially inhomogeneous dielectrics. IEEE Trans. Antennas Propag. AP-17: 667-669

Amin M B, James J R 1981 Techniques for the utilization of hexagonal ferrites in radar absorbers, Part I. Radio Electron. Eng. 51: 209-218

Aoto T, Yoshida N, Fukai I 1987 Transient analysis of the electromagnetic field for a wave absorber in three-dimensional space. IEEE Trans. Electromagn. Compat. EMC-29: 18-23 
Army Material Development and Readiness Command 1982 Radiation-resistant radar materials: Analytical and experimental study identifies materials potentially resistant to nuclear radiation damage. NTIS Tech Note, PB82970294XSP.

Arsaev I E 1982 Plane wave scattering by bodies of revolution. Radiotech. Electron. 27: 2101-2109

Ashley S, Gilmore C P 1988 Stealth. Pop. Sci. (7): 46-51

Baker D E, van der Neut C A 1988 Reflection measurements of microwave absorbers. Microwave $J .31: 95-98$

Bastiere A 1989 Decision-making aid for multi-layer radar absorbent coverings. Tech. Rep. ESA-TT-1173, European Space Agency, Paris

Bhattacharyya A K 1990 Radar cross section reduction of a flat plate by RAM coating. Microwave Opt. Technol. Lett. 3: 324-327

Bhattacharyya A K, Sengupta D L 1991 Radar cross section analysis and control (Norwood MA: Artech House)

Bhattacharyya A K, Tandon S K 1984 Radar cross section of a finite planar structure coated with a lossy dielectric. IEEE Trans. Antennas Propag. AP-32: 1003-1007

Blore W E 1964 The radar cross section of polyfoam towers. IEEE Trans. Antennas Propag. AP-12: 237-238

Bostick G 1985 Damping spurious microwave responses with absorbing materials. $E M C$ Technol. 14(2): 21-27

Bowman J J 1968 Effects of absorbers. In Methods of radar cross-section analysis(eds) J R Crispin Jr, K M Siegel (New York: Academic Press)

Bowman J J, Weston V H 1966 The effect of curvature on the reflection coefficient of layered absorbers. IEEE Trans. Antennas Propag. AP-14: 760-767

Bradshaw P S 1989 Signature management and structural materials. In Materials and processing-Move to the 90's, Proc. of SAMPE (Amsterdam: Elsevier Science) pp 187-196

Brown A 1992 Fundamentals of stealth design. Lockheed Horizons 31(8): 6-12

Brumley S 1987 Better RCS data with anechoic absorber characterization. Micro. RF 26: 143-148

Cain R N, Corda A J 1991 Active radar stealth device. Patent 5036 323, Dept. of the Navy, Washington DC

Cheng Y B, Ostertag E L 1986 An absorber-wall parallel-plate waveguide. IEEE Trans. Microwave Theory Tech. MTT-34: 761-766

Cherepanov A K 1974 Reflection of electromagnetic waves from an absorptive spiky surface. Radio Eng. Electron. Phys. 19: 120-123

Chou R, Ling H, Lee S W 1987 Reduction of the radar cross section of arbitrarily shaped cavity structures. Tech. Rep. NASACR 180307, Illinois Univ., Urbana-Champaign

Chou R-C 1988 Modal attenuation in multilayered coated waveguides. IEEE Trans. Microwave Theory Tech MTT-36: 1167-1176

Cobucci F 1991 Building air superiority. Aerosp. Mater. Compos. 3: 16-19

Curran J 1993 HP radar/EW testing solutions. HP RF and Microwave Test Symp Bangalore

Davies P, Popplewell J, LLewellyn J P 1986 Microwave absorption in ferrofluid composites. IEEE Trans. Magn. MAG-22: 1131-1133

de Hoop A T 1981 Theorem on maximum absorption of electromagnetic radiation by a scattering object of bounded extend. Radio Sci. 16: 971 - 974

Deleuze C 1992 Radar absorbing materials. Chocs 6: $15 \cdots 29$

Emerson W H 1973 Electromagnetic wave absorbers, anechoic chambers through the years. IEEE Trans. Antennas Propag. AP-21: 484--490

Engheta N, Zablocky P G 1990 A step towards determining transient response of chiral materials - Kramers-Kronig relations for chiral parameters. Electron. Lett. 26: 2132-2134

Falkenbach G J 1965 Limitations in determining absorbing material parameters. Proc. IEEE 53: 1097-1098

Fante R L, McCormack M T 1988 Reflection properties of the Salisbury screen. IEEE Trans. Antennas Propag. AP-36: 1443-1454

Fernandez A, Valenzula A 1985 General solution for single-layer electromagnetic wave absorber. Electron. Lett. 21: 20-21

Gauss A 1982 A new method of EM absorbing coating. Tech. Rep., AD A117472, Ballistic Research Lab., Aberdeen Proving Ground, MD 
Ginzton E L 1957 Microwave measurements (New York: McGraw Hill)

Guillot T 1992 Contribution to the modelling of the electromagnetic properties of random dielectric-conductor mixtures. Ph D thesis (Rep. ETN-93-93046), Office National d'Etudes et de Recherches Aerospatiales, Paris

Guillot T, Bobillot G 1991 Microwave measurement of the electrical conductivity of an elementary grain of a conducting powder. ONERA Tech. Rep. TP 1991-40 Paris

Hahn H T 1991 The variation of permeability with ferrite file density. J. Appl. Phys. B69:6195-6197

Halpren O, Johnson M J Jr Radar summary report of Harp project. OSRD Div 14, vol. 1 (part $\pi$ ), ch. $9-12$

Hanson R L, Kiehle M H 1982 Performance considerations in the design of subsonic missile. AI AA Aerosp. Sci. 20th Meeting (Paper No. 82-0371)

Harrington J J 1987 Missile decoy radar cross section enhancer. Patent NTIS ADD0135608XSP, Department of the Air Force. Washington DC

Hatakeyama K, Inui T 1984 Electromagnetic wave absorber using ferrite absorbing material dispersed with short metal fibers. IEEE Trans. Mayn. MAG-20: 1261-1263

He J, Lu Z, Su Y 1992 Experimental investigation on the ultra-wideband radar characteristics of coating RAMs targets. IEE Proc. Int. Conf. London: pp. 493496

Hemmati H, Mathur J C, Eichhorn W L 1985 Submillimeter and millimeter wave characterization of absorbing materials. Appl. Opt. 24: 4489-4492

Hempel K A, Roos W 1981 Microwave absorption along minor hysterisis loops of single domain particles with uniaxial magnetic anisotropy. IEEE Trans. Magn. MAG-17: 2642-2644

Holland R, Cho K S 1986 Radar cross-section of damped cylinders and dielectric strips. Tech. Rep. APITR 129 (Applied Physics Inc. Albuquerque NM)

Hurmuth H F 1983 On the effect of absorbing materials on electromagnetic waves with large relative bandwidth. IEEE Trans. Electromagn. Compat. EMC-25: 32-39

Jaggard D L, Engheta N 1989 Chirosorb as an invisible medium. Electron. Lett. 25: 173-174

Jaggard D L, Engheta N, Liu J 1990 Chiroshield - a Salisbury/Dallenbach shield alternative. Electron. Lett. 26: 1332-1334

Jaggard D L, Liu J C, Sun X 1991 Spherical chiroshield. Electron. Lett. 27: 77-79

Jones A K, Wooding E R 1964 A multilayer microwave absorber. IEEE Trans. Antennas Propag. AP-12: $508-509$

Joseph P J 1988 U TD (Uniform geometrical theory of diffraction) scattering analysis of pyramidal absorber for design of compact range chambers. Master's thesis (AFITCINR88193), Air Force Inst. of Technol., Wright-Patterson AFB OH

Kashiwa T, Yoshida N, Fukai I 1990 Simulation of the reduction characteristics of scattering from an aircraft coated with a thin-type absorber by the spatial network method. Electron. Lett. 26: $289-290$

Kent B 1982 An automated dual horn-reflector microwave absorber measurement system. Tech. Rep. AFWALTR811284 (Air Force Wright Aeronautical Labs Wright-Patterson AFB, OH.) Vol I

Knott E F 1979 The thickness criterion for single layer radar absorbers. IEEE Trans. Antennas Propag. AP-27: 698 701

Knott E F, Shaeffer J F, Tuley M T 1985 Radar cross section (Norwood MA: Artech House)

Kong J A 1975 Theory of electromagnetic waves (New York: Wiley Interscience)

Kumar A 1987 Acetylene black-A single-layer microwave absorbers. Electron. Lett. 23: 184-185

Kumar P M 1994 EM design aspects of airborne radomes. Project Report, National Aerospace Laboratories, Bangalore

Kumar P M, Vinoy K J, Jha R M 1994 An indexed database of radome (1960-1993). NAL Project Document PD AL 9405, National Aerospace Laboratories, Bangalore

Lee C S, Lee S W, Chuang S L 1986 Normal modes in an overmoded circular waveguide coated with lossy materials. IEEE Trans. Microwave Theory Tech. MTT-34: 773-785

Lee S W, Lo Y T, Chuang S L, Lee C S 1985 Numerical methods for analyzing electromagnetic scattering. Semiann. Rep., NAS126176141, Illinois Univ., Urbana-Champaign

Lehto A, Tourinen J, Raisanen A 1991 Reflectivity of absorbers in 100-200 GHz range. Electron. Lett. 27: 1699-1700

Leontovich M A 1957 Appendix of diffraction, refraction and reflection of radio waves. Rep. AD 117276 (US Govt. Printing Press, Washington DC) 
Li H J, Farhat N H, Shen Y 1989 Radar cross section reduction by absorber covering. $J$. Electromagn. Waves Appl. 3: 219235

Lynnworth L C 1964 Audio frequency characterization of RAM. Proc. IEEE 52: 98-99

MacFarlane G G 1945 Radar camouflage research and development by the Germans. Tech. Rep. T1905 M/99 TRE

Macleod J B 1989 Modeling of camouflage netting for radar cross section analysis. Master's thesis (AFITGEENG89J2), School of Engineering Air Force Inst. of Technol., Wright-Patterson AFB OH

Maffioli F 1970 Constrained variable metric optimization of layered electromagnetic absorbers. Alta Freq. (Eng. Edn.) 39: 154164

Martin P W 1992 Development of F-117 stealth fighter. Lockheed Horizons 31: 18 -23

Marty V, Combes P-F, Borderies P 1992 Radar cross section of a rectangular waveguide array with complex load and covered with dielectric. La Rech. Aerosp. 4: 15.25

McCauley J W, Halpin Jr. B M. Hynes T, Eitelman S D 1980 Radar absorptive ferrite/resin composites from industrial effluent. Ceramic Eng. Sci. Proc. 1: $356 \cdots 369$

McCluggage W A 1987 Study of radar cross section (RCS) characteristics and their application in future weapon systems. Master's thesis (ETN8892081), RAF College, Cranwell

Mishra S R. Pavlasek J J F, Yazar M N 1982 Design criteria for costeffective broad band absorber-lined chambers for EMS measurements. IEEE Trans. Electromagn. Compat. EMC24: $12-19$

Mitsmakher M Iu 1980 Quality of modern anechoic chambers and radio wave absorbing materials. Antenny 28: 147-164

Montgomery C G 1957 Techniques of microwave measurements (New York: McGraw-Hill)

Montgomery C G. Dicke R H. Purcell E 1948 Principles of microwave circuits. Radiation Lab Series 8 (Boston. MA: Boston Technol.)

Moreland J, Peters L Jr 1966 The specular radar cross section of absorber coated bodies. IEEE Trans. Antennas Propag. AP-14: 799800

Musal H M. Hahn H T 1989 Thin layer electromagnetic absorber design. IEEE Trans. Magn.MAG-25: $3851-3853$

Musal H M, Smith D C 1990 Universal design chart for specular absorbers. IEEE Trans. Magn. MAG-26: 14621464

Naamlooze Vennootschap Machinerieen 1936 French Patent 802728

Nagasubramanian G, Distefano S, Liang R H 1990 Silicon containing electroconductive polymers, structures made therefrom. Patent Application Rep. PAT-APPL-7-479 485, (NASA, Pasadena CA)

Nagornov A I, Postnik ov A I, Vasilev V P. Gordeev V A 1978 Study of the absorption properties of resistive films aligned perpendicular to the waveguide axis. Radiofizika 21: 151-153

Naito Y 1970 Generalised Snock's limits in ferrite. Jpn. J. Phys.

Naito Y, Suetake K 1965 Construction of multilayer absorbing wall for microwaves. Electron. Commun. Jpn. 48(12): 112-121

Naito Y, Suetake K 1971 Application of ferrite to Electromagnetic wave absorber and its characteristics. IEEE Trans. Microwave Theory Tech. MTT-19: 65-72

Olmedo L 1992 Absorbing materials based on conductive polymers Chocs 6: 53-65

Ono M. Suzuki M 1967 Reflection and attenuation characteristics of multilayer absorber at oblique incidence. Electron. Commun. Jpn. 50(9): 8492

Ono M, Okokawa S, Suzuki M 1967 Fundamental characteristics of the microwave absorber. Yamagata Univ. Bull. (Eng.) 9: 569-579

Ono M, Ikuta A, Katagiri Y 1979 Synthesis of an electromagnetic wave absorber with good reflection characteristics at both normal and oblique incidence. Electron. Commun. Jpn. 62: 59-62

Ono M, Yokoto T. Shibuya T 1983 A practical method of measuring the scattering characteristics of the pyramidal absorbers. Electron. Commun. Jpn. 66: 63-71

Perini J, Cohen L_ S 1991 Design of radar absorbing materials for wide range of angles of incidence. IEEE Int. Symp. on Electromagn. Compat. (New York: IEEE) pp 418-424

Post E J 1962 Formal structure of electromagnetics (Amsterdam: North Holland)

Rogers S W 1986 Radar cross section prediction for coated perfect conductors with arbitrary geometries. Master's thesis (Rep. AFITCINR86105T). Air Force Inst. of Technol., WrightPatterson AFB, OH 
Ruck G T, Barrick D E, Stuart W D, Krichbaum C K 1970 Radar cross-section handbook (New York: Plenum) vol. 2

Rudduck R C, Yu C L 1974 Circular waveguide method of measuring reflection properties of absorber panels. IEEE Trans. Antennas Propag. AP-22: 251-256

Salisbury W W 1952 Absorbent body for electromagnetic waves. US Patent 2599944

Schade H A 1945 Schornsteinfeger US tech. mission to Europe. Tech. Rep. 90-45 AD-47746

Schmitman C, Warwick G 1990 Building the B-2. Flight Int. 139: 24-27

Severin A 1956 Nonreflecting absorbers for microwave radiation. IEEE Trans. Antennas Propag. AP-4: $385-392$

Shi Z, Ding C, Jia Y 1993 Effects of absorbent materials on the RCS of a partially coated scatterer. Microwave Opt. Technol. Lett. 6: 109-111

Shimizu Y, Suetake K 1969 Minimum thickness design of broadband absorbing wall. Electron. Commun. Jpn. 52-B(4): 90-97

Shneyderman Y A 1985 Radio-absorbing materials. Tech. Rep. NTIS Rep. ADA1574961XSP (Foreign Technol. Div.), Wright-Patterson AFB, OH

Stonier R A 1991 Stealth aircraft and technology from World War II to the Gulf. SAMPE J. 27(4): $9-17$

Strickel M A, Taflove A 1990 Time domain synthesis of broad band absorptive coatings for two dimensional conducting targets. IEEE Trans. Antennas Propag. AP-38: 1084-1091

Swarner W G, Peters L Jr 1963 Radar cross sections of dielectric or plasma coated conducting spheres and circular cylinders. IEEE Trans. Antennas Propag. AP-11: 558-569

Sweetman B 1982 The bomber that radar cannot see. New Sci. 93: 565-568

Sweetman B 1987 Stealth in service. Interavia 42: 39-40

Tretyakov S A, Oksanen M I 1991 Biisotropic layer as a polarization transformer. Tech. Rep., ISBN-951-22-0770-2, Electromagnetics Lab. Helsinki Univ. of Technology, Espoo, Finland

Tsuji K 1992 Low observability aperture design for expendable countermeasures devices. Patent Rep., Patent-5 083 128, Dept. of the Navy, Washington, DC

Veinger A I, Zabrodskii A G, Krasikov L A, Khorosheva N E 1990 Anomalous microwave absorption in magnetically filled low-molecular-weight rubbers. Am. Inst. Phys. 855-856

Vinoy K J, Jha R M 1994 Radar absorbing materials (RAM): a cross indexed bibliography (1956-1993). NAL Project Document PD AL 9404, National Aerospace Laboratories, Bangalore

Walkington J W, Huster L W 1979 Achieving effective radar cross section flight profiles on the B-1 aircraft. In Soc.Fli. Test Eng., Proc. 10th Annu. Symp. (Lancaster, CA: Soc. Flight Test Eng.)

Weston V H 1963 Theory of absorbers in scattering. IEEE Trans. Antennas Propag. AP-11: $578-584$

Wims P R, Palmer D D 1991 Nondestructive microwave scanning measurements for material property evaluation. Review of progress in quantitative nondestructive evaluation. Proc. 17th Annu. Rev. (New York: Plenum P.) A10: 551-558

Yang C F, Burnside W D, Rudduck R C 1992 A periodic moment method solution for TM scattering from lossy dielectric bodies with application to wedge absorber. IEEE Trans. Antennas Propag. AP-40: 652-660

Yee K S 1966 Numerical solution of initial boundary value problems involving Maxwell's equations in isotropic media. IEEE Trans. Antennas Propag. AP-14: 302-307

Yi P, Gan Y 1991 Investigation on microwave absorber with additive of metal coated carbon fiber. Acta Aeronăut. Astronăut. Sin. B12: 655-657

Yokoi H, Fukumaro H 1971 Low-sidelobe paraboloidal antenna with microwave absorbers. Electron. Commun. Jpn. 54: 34-39 